九州大学学術情報リポジトリ

Kyushu University Institutional Repository

\title{
UC hierarchy and monodromy preserving deformation
}

Tsuda, Teruhisa

Faculty of Mathematics, Kyushu University

ht tp://hdl. hand le. net/2324/16324

出版情報 : MI Preprint Series. 2010-7，2010-01-28. 九州大学大学院数理学研究院 バージョン：

権利関係 : 


\section{Preprint Series}

Kyushu University

The Global COE Program

Math-for-Industry Education \& Research Hub

\section{UC hierarchy and monodromy preserving deformation}

\section{Teruhisa Tsuda}

\section{2010-7}

( Received January 28, 2010 )

Faculty of Mathematics

Kyushu University

Fukuoka, JAPAN 


\title{
UC hierarchy and monodromy preserving deformation
}

\author{
Teruhisa Tsuda \\ Faculty of Mathematics, Kyushu University, \\ Moto-oka, Fukuoka 819-0395, Japan.
}

January 21,2010

\begin{abstract}
The UC hierarchy is an extension of the KP hierarchy, which possesses not only an infinite set of positive time evolutions but also that of negative ones. Through a similarity reduction we derive from the UC hierarchy a class of the Schlesinger systems including the Garnier system and the sixth Painlevé equation, which describes the monodromy preserving deformations of Fuchsian linear differential equations with certain spectral types. We also present a unified formulation of the above Schlesinger systems as a canonical Hamiltonian system whose Hamiltonian functions are polynomials in the canonical variables.
\end{abstract}

\section{Introduction}

This work is aimed to present a certain connection between infinite-dimensional integrable systems of soliton type and finite-dimensional integrable systems of isomonodromic type. The KP hierarchy is, undoubtedly, the most basic one among the former and is a series of nonlinear partial differential equations in infinitely many independent variables $\boldsymbol{x}=\left(x_{1}, x_{2}, x_{3}, \ldots\right)$ that are consistent with each other. It literally includes as the first nontrivial member the KP (KadomtsevPetviashvili) equation

$$
\frac{3}{4} \frac{\partial^{2} f}{\partial x_{2}^{2}}=\frac{\partial}{\partial x_{1}}\left(\frac{\partial f}{\partial x_{3}}-\frac{3}{2} f \frac{\partial f}{\partial x_{1}}-\frac{1}{4} \frac{\partial^{3} f}{\partial x_{1}^{3}}\right)
$$

which is a typical soliton equation. If we count the degree of variables as $\operatorname{deg} x_{n}=n$ and $\operatorname{deg} f=$ -2 , then both sides of (1.1) are equally homogeneous (of degree -6) as differential polynomials in $f$ with respect to $x_{n}$. Every equation of the KP hierarchy is known to be homogeneous, in fact. In this sense we may say that the KP hierarchy forms a homogeneous integrable system equipped with an infinite set of time evolutions of positive degree. The UC hierarchy, introduced in [Tsu04], is an infinite-dimensional integrable system which naturally generalizes the KP hierarchy by taking into account the negative time evolutions besides the positive ones while keeping its homogeneity. The independent variables of the UC hierarchy consist of two sets of infinitely many variables $\boldsymbol{x}$ and $\boldsymbol{y}=\left(y_{1}, y_{2}, y_{3}, \ldots\right)$ with their degrees given as deg $x_{n}=n$ and $\operatorname{deg} y_{n}=-n$. In this paper we show

2000 Mathematics Subject Classification 34M55, 37K10.

E-mail: tudateru@math.kyushu-u.ac.jp 
that a similarity reduction of the UC hierarchy yields a broad class of the Schlesinger systems including the Garnier system and the sixth Painlevé equation, which describes the monodromy preserving deformations of Fuchsian linear differential equations with certain spectral types.

We begin by recalling the definition of the UC hierarchy. Let us introduce the commuting pair of linear differential operators (called the vertex operators)

$$
\begin{aligned}
X^{ \pm}(z) & =\sum_{n \in \mathbb{Z}} X_{n}^{ \pm} z^{n}=e^{ \pm \xi\left(\boldsymbol{\xi}-\widetilde{\partial}_{y}, z\right)} e^{\mp \xi\left(\widetilde{\partial}_{x}, z^{-1}\right)}, \\
Y^{ \pm}(w) & =\sum_{n \in \mathbb{Z}} Y_{n}^{ \pm} w^{n}=e^{ \pm \xi\left(\boldsymbol{y}-\widetilde{\partial}_{x}, w\right)} e^{\mp \xi\left(\widetilde{\partial}_{y}, w^{-1}\right)},
\end{aligned}
$$

where we have used the notations

$$
\xi(\boldsymbol{x}, z)=\sum_{n=1}^{\infty} x_{n} z^{n} \quad \text { and } \quad \widetilde{\partial}_{x}=\left(\frac{\partial}{\partial x_{1}}, \frac{1}{2} \frac{\partial}{\partial x_{2}}, \frac{1}{3} \frac{\partial}{\partial x_{3}}, \ldots\right) .
$$

Definition 1.1. For an unknown function $\tau=\tau(x, y)$, the simultaneous bilinear equation

$$
\sum_{i+j=-1} X_{i}^{-} \tau \otimes X_{j}^{+} \tau=\sum_{i+j=-1} Y_{i}^{-} \tau \otimes Y_{j}^{+} \tau=0
$$

is called the UC hierarchy.

The UC hierarchy is homogeneous indeed as it has the following scaling symmetry: if $\tau$ is a solution of (1.3) then so is $\tau\left(c x_{1}, c^{2} x_{2}, \ldots, c^{-1} y_{1}, c^{-2} y_{2}, \ldots\right)$ for any $c \in \mathbb{C}^{\times}$. The UC hierarchy is regarded as an extension of the KP hierarchy. If $\tau$ does not depend on $y$, then the latter equality of (1.3) trivially holds and the former reduces to the bilinear expression of the KP hierarchy, which is due to Date-Jimbo-Kashiwara-Miwa (see [Kac90, MJD00]); for reference the variable transformation toward the original KP equation, (1.1), is given by $f=2\left(\partial / \partial x_{1}\right)^{2} \log \tau$. We always require the solution $\tau=\tau(\boldsymbol{x}, \boldsymbol{y})$, called the $\tau$-function, to be an entire function with respect to each independent variable. Note that $\tau$-functions are distinguished up to multiplication by constants, as can be seen from (1.3). Concerning the UC hierarchy there is a counterpart of the Sato theory about the KP hierarchy; cf. [Sat81]. That is, the totality of solutions of the UC hierarchy forms a direct product of two Sato Grassmannians and the action of its transformation group can be realized by means of the vertex operators. For details to [Tsu04]. Of particular interest is its homogeneous polynomial solution, which is a fixed solution with respect to the above scaling symmetry.

Let $\lambda=\left(\lambda_{1}, \lambda_{2}, \ldots, \lambda_{\ell}\right)$ and $\mu=\left(\mu_{1}, \mu_{2}, \ldots, \mu_{\ell^{\prime}}\right)$ be a pair of partitions. Consider the following determinant of twisted Jacobi-Trudi type:

$$
S_{[\lambda, \mu]}=\operatorname{det}\left(\begin{array}{ll}
\widetilde{h}_{\mu_{\ell^{\prime}-i+1}+i-j}, & i \leq \ell^{\prime} \\
h_{\lambda_{i-\ell^{\prime}}-i+j}, & i>\ell^{\prime}+1
\end{array}\right)_{1 \leq i, j \leq \ell+\ell^{\prime}},
$$

where $h_{n}=h_{n}(\boldsymbol{x})(n \in \mathbb{Z})$ is a polynomial in only $\boldsymbol{x}$ and is defined by the generating function

$$
e^{\xi(\boldsymbol{x}, z)}=\sum_{n \in \mathbb{Z}} h_{n} z^{n}
$$

and $\widetilde{h}_{n}=\widetilde{h}_{n}(\boldsymbol{y})$ is exactly the same as $h_{n}$ except replacing $\boldsymbol{x}$ with $\boldsymbol{y}$. If $\mu=\emptyset$ then (1.4) reduces to the (usual) Jacobi-Trudi formula: $S_{\lambda}=S_{[\lambda, \emptyset]}=\operatorname{det}\left(h_{\lambda_{i}-i+j}\right)$, which defines the Schur function 
$S_{\lambda}=S_{\lambda}(\boldsymbol{x})$. The polynomial $S_{[\lambda, \mu]}=S_{[\lambda, \mu]}(\boldsymbol{x}, \boldsymbol{y})$ is called the universal character and was originally introduced by Koike [Koi89] in the study of classical groups. It is easy to see that $S_{[\lambda, \mu]}$ becomes a homogeneous polynomial whose degree equals the difference $|\lambda|-|\mu|$, where the sum $|\lambda|=$ $\lambda_{1}+\cdots+\lambda_{\ell}$ denotes the weight of a partition $\lambda$. A few examples are $S_{[\emptyset, \emptyset]}=1, S_{[(1), \emptyset]}=x_{1}$, $S_{[(1),(1)]}=x_{1} y_{1}-1, S_{[(2,1),(1)]}=y_{1}\left(x_{1}{ }^{3} / 3-x_{3}\right)-x_{1}{ }^{2}$, etc. Remarkably, the set of homogeneous polynomial solutions of the UC hierarchy, (1.3), coincides with that of the universal characters $\left\{S_{[\lambda, \mu]}(\boldsymbol{x}, \boldsymbol{y})\right\}_{\lambda, \mu \text { :partitions. }}$.

By considering general homogeneous solutions of the UC hierarchy that are not necessarily polynomials, we can find a link to the theory of monodromy preserving deformations. Let us explain it in more detail. First we derive from the original one, (1.3), similar bilinear equations among solutions generated by successive application of vertex operators. Let $\tau_{m, n}=\tau_{m, n}(\boldsymbol{x}, \boldsymbol{y})$ denote such a sequence of solutions of the UC hierarchy. A typical example of the bilinear equations is

$$
\tau_{m, n} \otimes \tau_{m+1, n+1}=\sum_{i+j=0} X_{i}^{-} \tau_{m+1, n} \otimes X_{j}^{+} \tau_{m, n+1}
$$

Next we impose on the sequence $\tau_{m, n}$ of solutions homogeneity

$$
E \tau_{m, n}=d_{m, n} \tau_{m, n} \quad\left(d_{m, n} \in \mathbb{C}\right)
$$

and periodicity

$$
\tau_{m+L, n}=\tau_{m, n+L}=\tau_{m, n}
$$

for an integer $L(\geq 2)$ fixed. Here we have used the Euler operator

$$
E=\sum_{n=1}^{\infty}\left(n x_{n} \frac{\partial}{\partial x_{n}}-n y_{n} \frac{\partial}{\partial y_{n}}\right)
$$

which is a linear differential operator measuring the degree of a homogeneous function; for instance, $E S_{[\lambda, \mu]}=(|\lambda|-|\mu|) S_{[\lambda, \mu]}$. Finally we substitute into each $x_{n}$ and $y_{n}$ the 'power sum' of new independent variables $\boldsymbol{t}=\left(t_{0}, t_{1}, \ldots, t_{N}\right)$ as

$$
x_{n}=\frac{1}{n} \sum_{i=0}^{N} \theta_{i} t_{i}^{n} \quad \text { and } \quad y_{n}=\frac{1}{n} \sum_{i=0}^{N} \theta_{i} t_{i}^{-n} \quad(n=1,2, \ldots)
$$

where $\theta_{i} \in \mathbb{C}$ are constant parameters. In view of the homogeneity (1.5), we may take $t_{0}=1$ without loss of generality. Under the reduction conditions (1.5), (1.6), and (1.7), the UC hierarchy yields a system of nonlinear partial differential equations in $N$ variables, hereafter denoted by $\mathcal{G}_{L, N}$, whose phase space is essentially of $2 N(L-1)$ dimension. To sum up the above procedure, we say that $\mathcal{G}_{L, N}$ is a similarity reduction of the UC hierarchy. The system $\mathcal{G}_{L, N}$ is a finite-dimensional integrable system of isomonodromic type. For instance $\mathcal{G}_{2, N}$ corresponds to the Garnier system in $N$ variables and $\mathcal{G}_{2,1}$, the first nontrivial case, does the sixth Painlevé equation. From the viewpoint of the UC hierarchy we can clearly understand various aspects of $\mathcal{G}_{L, N}$, e.g., Hirota bilinear relations for $\tau$-functions, Weyl group symmetries, and algebraic solutions expressed in terms of the universal character.

As analogous to the case of the KP hierarchy, the UC hierarchy (1.3) generates the linear equations for unknown functions (called the wave functions)

$$
\psi_{m, n}=\psi_{m, n}(\boldsymbol{x}, \boldsymbol{y}, k)=\frac{\tau_{m, n-1}\left(\boldsymbol{x}-\left[k^{-1}\right], \boldsymbol{y}-[k]\right)}{\tau_{m, n}(\boldsymbol{x}, \boldsymbol{y})} e^{\xi(\boldsymbol{x}, k)},
$$


where $[k]=\left(k, k^{2} / 2, k^{3} / 3, \ldots\right)$. Through the reduction procedure they induce an auxiliary system of linear differential equations; one of which is a Fuchsian system of rank $L$ in the spectral variable $z=k^{L}$ with $N+3$ poles on the Riemann sphere, and the others govern its monodromy preserving deformations. The nonlinear system $\mathcal{G}_{L, N}$ can be reformulated as a compatibility condition of this auxiliary linear system (Lax formalism). Remark here that the compatibility itself is a priori established because all the linear equations originate from the same bilinear equation (1.3).

The spectral type of the Fuchsian system under consideration is given by the $(N+3)$-tuple

$$
\underbrace{(L-1,1), \ldots,(L-1,1)}_{N+1},(1,1, \ldots, 1),(1,1, \ldots, 1)
$$

of partitions of $L$, which indicates how the characteristic exponents overlap at each of the $N+3$ singularities. Thus we conclude that $\mathcal{G}_{L, N}$ is equivalent to a particular case of the Schlesinger systems specified by this spectral type. We also present a unified description of $\mathcal{G}_{L, N}$ for any $L$ and $N$ as a canonical Hamiltonian system, denoted by $\mathcal{H}_{L, N}$, whose Hamiltonian functions are polynomials in the canonical variables.

In the next section we derive some difference (and differential) equations from the UC hierarchy as a preliminary. In Sect. 3, we construct a sequence of homogeneous solutions of the UC hierarchy and present its Weyl group symmetry of type $A$. In Sect. 4, we consider a similarity reduction of the UC hierarchy by requiring its solutions to satisfy the homogeneity and periodicity. As a result we obtain a nonlinear system $\mathcal{G}_{L, N}$ of partial differential equations, which provides an extension of both the Garnier system and the sixth Painlevé equation. The universal characters $S_{[\lambda, \mu]}$ are homogeneous solutions of the UC hierarchy and thereby consistent with the similarity reduction. Hence, as described in Sect. 5, it is immediate to obtain particular solutions of $\mathcal{G}_{L, N}$ expressed in terms of $S_{[\lambda, \mu]}$. The subject of Sect. 6 is the Lax formalism of the systems $\mathcal{G}_{L, N}$, which reveals that they constitute a class of the Schlesinger systems. We show that the auxiliary linear problem of $\mathcal{G}_{L, N}$ arises naturally from the linear equations satisfied by the wave functions of the UC hierarchy. In Sect. 7, we transform $\mathcal{G}_{L, N}$ into the canonical Hamiltonian system $\mathcal{H}_{L, N}$ with polynomial Hamiltonian functions. Section 8 is devoted to the birational symmetries. We observe that the Weyl group actions, discussed in Sect. 3, give rise to birational canonical transformations of $\mathcal{H}_{L, N}$. In the appendix we briefly indicate a relationship between our polynomial Hamiltonian structure and that given by Kimura and Okamoto [KO84] for the Garnier system, i.e., the case where $L=2$.

\section{Method for generating a 'closed' functional equation}

Unlike in the case of the KP hierarchy, every differential equation of the UC hierarchy with respect to the original variables $\boldsymbol{x}$ and $\boldsymbol{y}$ is of infinite order. In this section we show how to overcome this difficulty, i.e., a method for generating a 'closed' functional equation from the UC hierarchy; cf. [DJM82].

We first recall that if $\tau=\tau(\boldsymbol{x}, \boldsymbol{y})$ is a solution of (1.3) then so are $X^{+}(a) \tau$ and $Y^{+}(b) \tau$ for any $a, b \in \mathbb{C}^{\times}$. With this fact in mind, let us take our interest in bilinear equations for a sequence of solutions generated by successive application of vertex operators. Suppose $\tau_{0,0}=\tau(\boldsymbol{x}, \boldsymbol{y})$ to be a 
solution of the UC hierarchy, (1.3). Define a sequence $\tau_{m, n}$ of solutions by

$$
\tau_{m, n}=\prod_{i=0}^{m-1} X^{+}\left(a_{i}\right) \prod_{j=0}^{n-1} Y^{+}\left(b_{j}\right) \tau_{0,0},
$$

where we write as

$$
\prod_{i=0}^{m-1} X^{+}\left(a_{i}\right)=X^{+}\left(a_{m-1}\right) \cdots X^{+}\left(a_{1}\right) X^{+}\left(a_{0}\right) .
$$

Then we can derive similar bilinear equations from the UC hierarchy, the original one (1.3).

Lemma 2.1. For integers $m, n \geq 0$, it holds that

$$
\begin{gathered}
\sum_{i+j=-m-1} X_{i}^{-} \tau_{0,0} \otimes X_{j}^{+} \tau_{m, n}=\sum_{i+j=-n-1} Y_{i}^{-} \tau_{0,0} \otimes Y_{j}^{+} \tau_{m, n}=0 \\
\tau_{0,0} \otimes \tau_{1, n}-\sum_{i+j=0} X_{i}^{-} \tau_{1,0} \otimes X_{j}^{+} \tau_{0, n}=\sum_{i+j=-n-1} Y_{i}^{-} \tau_{1,0} \otimes Y_{j}^{+} \tau_{0, n}=0 \\
\sum_{i+j=-m-1} X_{i}^{-} \tau_{0,1} \otimes X_{j}^{+} \tau_{m, 0}=\tau_{0,0} \otimes \tau_{m, 1}-\sum_{i+j=0} Y_{i}^{-} \tau_{0,1} \otimes Y_{j}^{+} \tau_{m, 0}=0
\end{gathered}
$$

Proof. Notice that the operators $X_{i}^{ \pm}(i \in \mathbb{Z})$ satisfy the fermionic relations: $X_{i}^{ \pm} X_{j}^{ \pm}+X_{j-1}^{ \pm} X_{i+1}^{ \pm}=0$ and $X_{i}^{+} X_{j}^{-}+X_{j+1}^{-} X_{i-1}^{+}=\delta_{i+j, 0}$. The same relations hold also for $Y_{i}^{ \pm}$. Moreover, $X_{i}^{ \pm}$and $Y_{j}^{ \pm}$mutually commute. See [Tsu04]. By virtue of the above relations, applying $1 \otimes \prod_{i=0}^{m-1} X^{+}\left(a_{i}\right) \prod_{j=0}^{n-1} Y^{+}\left(b_{j}\right)$, $X^{+}\left(a_{0}\right) \otimes \prod_{j=0}^{n-1} Y^{+}\left(b_{j}\right)$, and $Y^{+}\left(b_{0}\right) \otimes \prod_{i=0}^{m-1} X^{+}\left(a_{i}\right)$ to (1.3), we obtain (2.2), (2.3), and (2.4), respectively.

We shall look closely at (2.2), which corresponds to the original UC hierarchy (1.3) when $m=n=0$. It can be rewritten equivalently into

$$
\begin{aligned}
& \frac{1}{2 \pi \sqrt{-1}} \oint z^{m} e^{\xi\left(\boldsymbol{x}-\boldsymbol{x}^{\prime}, z\right)} \mathrm{d} z \tau_{0,0}\left(\boldsymbol{x}^{\prime}+\left[z^{-1}\right], \boldsymbol{y}^{\prime}+[z]\right) \tau_{m, n}\left(\boldsymbol{x}-\left[z^{-1}\right], \boldsymbol{y}-[z]\right)=0, \\
& \frac{1}{2 \pi \sqrt{-1}} \oint w^{n} e^{\xi\left(\boldsymbol{y}-\boldsymbol{y}^{\prime}, w\right)} \mathrm{d} w \tau_{0,0}\left(\boldsymbol{x}^{\prime}+[w], \boldsymbol{y}^{\prime}+\left[w^{-1}\right]\right) \tau_{m, n}\left(\boldsymbol{x}-[w], \boldsymbol{y}-\left[w^{-1}\right]\right)=0
\end{aligned}
$$

with $\boldsymbol{x}, \boldsymbol{y}, \boldsymbol{x}^{\prime}$, and $\boldsymbol{y}^{\prime}$ being arbitrary parameters, where $\oint \frac{\mathrm{d} z}{2 \pi \sqrt{-1}}$ means taking the coefficient of $1 / z$ of the integrand as a (formal) Laurent series expansion in $z$. If we try to write down a differential equation naively after the case of the KP hierarchy, namely if we consider the Taylor series expansion of (2.5a) around $\left\{\boldsymbol{x}^{\prime}=\boldsymbol{x}, \boldsymbol{y}^{\prime}=\boldsymbol{y}\right\}$, then we have an infinite set of differential equations of infinite order; see [Tsu04]. This result reflects the fact that the integrand of (2.5a) under the substitution $\boldsymbol{x}^{\prime}=\boldsymbol{x}$ and $\boldsymbol{y}^{\prime}=\boldsymbol{y}$ may admit an essential singularity not only at $z=0$ but also at $z=\infty$. However, we can construct a functional equation in a closed expression by taking an appropriate choice of parameters $\boldsymbol{x}, \boldsymbol{y}, \boldsymbol{x}^{\prime}$, and $\boldsymbol{y}^{\prime}$ instead.

Let $I, J \subset \mathbb{Z}$ be a disjoint pair of finite indexing sets. By specializing the parameters in (2.5) as

$$
\boldsymbol{x}^{\prime}=\boldsymbol{x}-\sum_{j \in I}\left[t_{j}\right]+\sum_{j \in J}\left[t_{j}\right], \quad \boldsymbol{y}^{\prime}=\boldsymbol{y}-\sum_{j \in I}\left[t_{j}^{-1}\right]+\sum_{j \in J}\left[t_{j}^{-1}\right],
$$




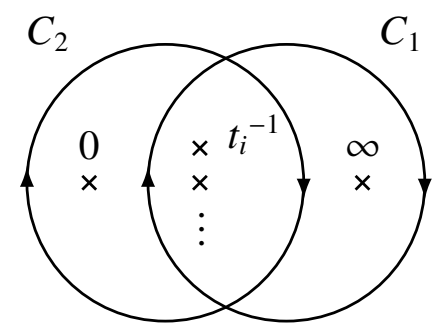

$(i \in I)$

Figure 1: Contours of integration and singularities of $F(z)$.

we obtain

$$
\begin{aligned}
& \Omega_{1}:=z^{m} e^{\xi\left(\boldsymbol{x}-\boldsymbol{x}^{\prime}, z\right)} \mathrm{d} z=z^{m} \frac{\prod_{j \in J}\left(1-t_{j} z\right)}{\prod_{j \in I}\left(1-t_{j} z\right)} \mathrm{d} z \quad\left(\left|t_{j} z\right|<1\right), \\
& \Omega_{2}:=w^{n} e^{\xi\left(\boldsymbol{y}-\boldsymbol{y}^{\prime}, w\right)} \mathrm{d} w=w^{n} \frac{\prod_{j \in J}\left(1-w / t_{j}\right)}{\prod_{j \in I}\left(1-w / t_{j}\right)} \mathrm{d} w \quad\left(\left|w / t_{j}\right|<1\right) .
\end{aligned}
$$

Here we have used the Taylor expansion, $\log (1-u)=-\sum_{k=1}^{\infty} u^{k} / k$ valid for $|u|<1$. Suppose $z w=1$. Then we observe that

$$
\Omega_{2}=z^{-n} \frac{\prod_{j \in J}\left(1-1 / t_{j} z\right)}{\prod_{j \in I}\left(1-1 / t_{j} z\right)}\left(-\frac{\mathrm{d} z}{z^{2}}\right)=-z^{|I|-|J|-m-n-2} \frac{\prod_{j \in I}\left(-t_{j}\right)}{\prod_{j \in J}\left(-t_{j}\right)} \Omega_{1} .
$$

Consequently, both integrands of (2.5a) and (2.5b) coincide up to constant multiplication if the condition $|I|-|J|=m+n+2$ is fulfilled. In this case the integrand of (2.5a) reads

$$
F(z)=z^{m} \frac{\prod_{j \in J}\left(1-t_{j} z\right)}{\prod_{j \in I}\left(1-t_{j} z\right)} \tau_{0,0}\left(\boldsymbol{x}^{\prime}+\left[z^{-1}\right], \boldsymbol{y}^{\prime}+[z]\right) \tau_{m, n}\left(\boldsymbol{x}-\left[z^{-1}\right], \boldsymbol{y}-[z]\right) .
$$

Since $\tau_{0,0}(\boldsymbol{x}, \boldsymbol{y})$ and $\tau_{m, n}(\boldsymbol{x}, \boldsymbol{y})$ are entire, $F(z)$ has the $|I|+2$ singularities: $z=1 / t_{i}$ (simple poles) for $i \in I$ and $z=0, \infty$ (which may be essential singularities). Hence (2.5) becomes

$$
\int_{C_{1}} F(z) \mathrm{d} z=\int_{C_{2}} F(z) \mathrm{d} z=0
$$

where the integration contour $C_{1}$ (resp. $C_{2}$ ) is a positively oriented small circle around $z=0$ (resp. $z=\infty$ ) such that all the other singularities are exterior to it; see Figure 1. We verify through the Cauchy-Goursat theorem that

$$
\sum_{i \in I} \operatorname{Res}_{z=1 / t_{i}} F(z) \mathrm{d} z=0
$$

by canceling contribution of residues at $z=\infty$ and $z=0$ respectively to the first and second integrals in (2.6). In other words, we have successfully avoided the residue calculus at possible essential singularities $z=0, \infty$ thanks to the presence of $t w o$ bilinear equations (2.5a) and (2.5b).

Now we prepare some notations. For a function $f=f(\boldsymbol{x}, \boldsymbol{y})$, we define a shift operator $T_{i}$ by $T_{i}(f)=f\left(\boldsymbol{x}-\left[t_{i}\right], \boldsymbol{y}-\left[t_{i}^{-1}\right]\right)$. We also write $T_{\left\{i_{1}, i_{2}, \ldots, i_{r}\right\}}(f)=T_{i_{1}} \circ T_{i_{2}} \circ \cdots \circ T_{i_{r}}(f)$ for brevity. Then (2.7) takes the following form:

$$
\sum_{i \in I} t_{i}^{n} \frac{\prod_{j \in J}\left(t_{i}-t_{j}\right)}{\prod_{j \in I \backslash\{i\}}\left(t_{i}-t_{j}\right)} T_{I \backslash\{i\}}\left(\tau_{0,0}\right) T_{J \cup\{i\}}\left(\tau_{m, n}\right)=0,
$$


which can be regarded as a difference equation with each $t_{i}$ being the difference interval. Along the same lines as (2.2), also (2.3) and (2.4) generate similar difference equations. Summarizing above we have the

Proposition 2.2. The following difference equations hold.

1. If $|I|-|J|=m+n+2$ and $m, n \geq 0$, then

$$
\sum_{i \in I} t_{i}^{n} \frac{\prod_{j \in J}\left(t_{i}-t_{j}\right)}{\prod_{j \in I \backslash\{i\}}\left(t_{i}-t_{j}\right)} T_{I \backslash\{i\}}\left(\tau_{0,0}\right) T_{J \cup\{i\}}\left(\tau_{m, n}\right)=0 .
$$

2. If $|I|-|J|=n+1$ and $n \geq 0$, then

$$
T_{I}\left(\tau_{0,0}\right) T_{J}\left(\tau_{1, n}\right)=\sum_{i \in I} \frac{\prod_{j \in J}\left(1-t_{j} / t_{i}\right)}{\prod_{j \in I \backslash\{i\}}\left(1-t_{j} / t_{i}\right)} T_{I \backslash\{i\}}\left(\tau_{1,0}\right) T_{J \cup\{i\}}\left(\tau_{0, n}\right) .
$$

3. If $|I|-|J|=m+1$ and $m \geq 0$, then

$$
T_{I}\left(\tau_{0,0}\right) T_{J}\left(\tau_{m, 1}\right)=\sum_{i \in I} \frac{\prod_{j \in J}\left(1-t_{i} / t_{j}\right)}{\prod_{j \in I \backslash\{i\}}\left(1-t_{i} / t_{j}\right)} T_{I \backslash\{i\}}\left(\tau_{0,1}\right) T_{J \cup\{i\}}\left(\tau_{m, 0}\right) .
$$

Example 2.3. Consider the case $m=n=0, I=\{1,2,3\}$, and $J=\{4\}$. Write $\tau=\tau_{0,0}$. Then (2.8) reduces to the equation

$$
\begin{gathered}
\left(t_{1}-t_{2}\right)\left(t_{3}-t_{4}\right) T_{1,2}(\tau) T_{3,4}(\tau)+\left(t_{2}-t_{3}\right)\left(t_{1}-t_{4}\right) T_{2,3}(\tau) T_{1,4}(\tau) \\
+\left(t_{3}-t_{1}\right)\left(t_{2}-t_{4}\right) T_{1,3}(\tau) T_{2,4}(\tau)=0
\end{gathered}
$$

which was found by Ohta [Oht07] as a quadratic relation for the universal character.

Let $m=1, n=0, I=\{1,2,3\}$, and $J=\emptyset$. Then (2.8) reduces to

$$
\left(t_{1}-t_{2}\right) T_{1,2}\left(\tau_{0,0}\right) T_{3}\left(\tau_{1,0}\right)+\left(t_{2}-t_{3}\right) T_{2,3}\left(\tau_{0,0}\right) T_{1}\left(\tau_{1,0}\right)+\left(t_{3}-t_{1}\right) T_{1,3}\left(\tau_{0,0}\right) T_{2}\left(\tau_{1,0}\right)=0 .
$$

Let $n=1, I=\{1,2\}$, and $J=\emptyset$. Then (2.9) reduces to

$$
\left(t_{1}-t_{2}\right) T_{1,2}\left(\tau_{0,0}\right) \tau_{1,1}=t_{1} T_{1}\left(\tau_{0,1}\right) T_{2}\left(\tau_{1,0}\right)-t_{2} T_{2}\left(\tau_{0,1}\right) T_{1}\left(\tau_{1,0}\right) .
$$

The above difference equations, (2.11) and (2.12), were introduced in a study of the connection between the universal character and $q$-Painlevé equations; see [Tsu05b, Tsu09a].

Furthermore, we can obtain a functional equation that involves derivative terms from the difference equations through a limit process causing a confluence of the poles $z=1 / t_{i}$. For instance let us take the limit $t_{3} \rightarrow t_{1}$ in (2.11). Rewrite $(t, s)=\left(t_{1}, t_{2}\right)$ and shift the variables as $\boldsymbol{x} \mapsto \boldsymbol{x}+[t]$ and $\boldsymbol{y} \mapsto \boldsymbol{y}+\left[t^{-1}\right]$. Then we find

$$
\begin{aligned}
\left(D_{\delta_{t}}\right. & \left.+\frac{t}{s-t}\right) \tau_{0,0}\left(\boldsymbol{x}-[s], \boldsymbol{y}-\left[s^{-1}\right]\right) \cdot \tau_{1,0}(\boldsymbol{x}, \boldsymbol{y}) \\
& +\frac{t}{t-s} \tau_{0,0}\left(\boldsymbol{x}-[t], \boldsymbol{y}-\left[t^{-1}\right]\right) \tau_{1,0}\left(\boldsymbol{x}+[t]-[s], \boldsymbol{y}+\left[t^{-1}\right]-\left[s^{-1}\right]\right)=0 .
\end{aligned}
$$


Here we have introduced the vector fields

$$
\delta_{t}=\sum_{n=1}^{\infty}\left(t^{n} \frac{\partial}{\partial x_{n}}-t^{-n} \frac{\partial}{\partial y_{n}}\right) \quad \text { and } \quad \tilde{\delta}_{t}=\sum_{n=1}^{\infty}\left(n t^{n} \frac{\partial}{\partial x_{n}}+n t^{-n} \frac{\partial}{\partial y_{n}}\right),
$$

and let $D_{v}$ denote the Hirota differential with respect to a vector field $\boldsymbol{v}$. If we take continuously the limit $s \rightarrow t$ in (2.13) with divided by $t-s$, then we obtain

$$
\left(D_{\delta_{t}}^{2}-D_{\delta_{t}}+D_{\tilde{\delta}_{t}}\right) \tau_{0,0}\left(\boldsymbol{x}-[t], \boldsymbol{y}-\left[t^{-1}\right]\right) \cdot \tau_{1,0}(\boldsymbol{x}, \boldsymbol{y})=0 .
$$

In this manner we can produce various functional equations from the UC hierarchy. We list the ones relevant to the following sections.

Proposition 2.4. The following difference (and differential) equations hold:

$$
\begin{aligned}
& (t-s) \tau_{m, n}\left(\boldsymbol{x}-[t]-[s], \boldsymbol{y}-\left[t^{-1}\right]-\left[s^{-1}\right]\right) \tau_{m+1, n+1}(\boldsymbol{x}, \boldsymbol{y}) \\
& \quad-t \tau_{m, n+1}\left(\boldsymbol{x}-[t], \boldsymbol{y}-\left[t^{-1}\right]\right) \tau_{m+1, n}\left(\boldsymbol{x}-[s], \boldsymbol{y}-\left[s^{-1}\right]\right) \\
& \quad+s \tau_{m, n+1}\left(\boldsymbol{x}-[s], \boldsymbol{y}-\left[s^{-1}\right]\right) \tau_{m+1, n}\left(\boldsymbol{x}-[t], \boldsymbol{y}-\left[t^{-1}\right]\right)=0 \\
& \left(D_{\delta_{t}}-1\right) \tau_{m, n+1}(\boldsymbol{x}, \boldsymbol{y}) \cdot \tau_{m+1, n}(\boldsymbol{x}, \boldsymbol{y}) \\
& \quad+\tau_{m, n}\left(\boldsymbol{x}-[t], \boldsymbol{y}-\left[t^{-1}\right]\right) \tau_{m+1, n+1}\left(\boldsymbol{x}+[t], \boldsymbol{y}+\left[t^{-1}\right]\right)=0 \\
& \left(D_{\delta_{t}}+\frac{t}{s-t}\right) \tau_{m, n}\left(\boldsymbol{x}-[s], \boldsymbol{y}-\left[s^{-1}\right]\right) \cdot \tau_{m+1, n}(\boldsymbol{x}, \boldsymbol{y}) \\
& \quad+\frac{t}{t-s} \tau_{m, n}\left(\boldsymbol{x}-[t], \boldsymbol{y}-\left[t^{-1}\right]\right) \tau_{m+1, n}\left(\boldsymbol{x}+[t]-[s], \boldsymbol{y}+\left[t^{-1}\right]-\left[s^{-1}\right]\right)=0 .
\end{aligned}
$$

Proof. Clearly (2.15) and (2.17) are equivalent to (2.12) and (2.13), respectively. Taking the limit $s \rightarrow t$ in (2.15) leads to (2.16).

\section{Homogeneous $\tau$-sequence and its Weyl group symmetry}

This section is concerned with a sequence of homogeneous solutions of the UC hierarchy, connected by vertex operators. We show that such a sequence naturally admits a commutative pair of Weyl group actions of type $A$ generated by a permutation of two serial vertex operators.

We first introduce partial differential operators $V_{X}(c)$ and $V_{Y}\left(c^{\prime}\right)\left(c, c^{\prime} \in \mathbb{C}\right)$ defined by

$$
V_{X}(c)=\int_{\gamma} X^{+}(z) z^{-c-1} \mathrm{~d} z \quad \text { and } \quad V_{Y}\left(c^{\prime}\right)=\int_{\gamma^{\prime}} Y^{+}\left(z^{-1}\right) z^{c^{\prime}-1} \mathrm{~d} z,
$$

where the integration paths $\gamma, \gamma^{\prime}:[0,1] \rightarrow \mathbb{C}$ is taken such that $\left[X^{+}(z) z^{-c}\right]_{\gamma(0)}^{\gamma(1)}=\left[Y^{+}\left(z^{-1}\right) z^{c^{\prime}}\right]_{\gamma^{\prime}(0)}^{\gamma^{\prime}(1)}=0$. For instance $\gamma$ and $\gamma^{\prime}$ can be chosen to be cycles. Note that in general $\gamma$ and $\gamma^{\prime}$ may depend on $c$ and $c^{\prime}$, respectively. It is easy to see that $V_{X}(c)$ and $V_{Y}\left(c^{\prime}\right)$ mutually commute.

Suppose $\tau_{0,0}=\tau_{0,0}(\boldsymbol{x}, \boldsymbol{y})$ to be a solution of the UC hierarchy (1.3) satisfying the homogeneity $E \tau_{0,0}=d_{0,0} \tau$. Instead of (2.1), let us consider a sequence $\left\{\tau_{m, n}\right\}_{m, n \geq 0}$ determined recursively by

$$
\tau_{m+1, n}=V_{X}\left(c_{m}\right) \tau_{m, n} \quad \text { and } \quad \tau_{m, n+1}=V_{Y}\left(c_{n}^{\prime}\right) \tau_{m, n}
$$


for arbitrary constant parameters $c_{m}, c_{n}^{\prime} \in \mathbb{C}$ given. Since the UC hierarchy (1.3) takes the form of bilinear equations, it can be verified in exactly the same way as (2.1) that each $\tau_{m, n}$ gives a solution of (1.3). Furthermore, they all obey the homogeneity

$$
E \tau_{m, n}=d_{m, n} \tau_{m, n}
$$

with $d_{m+1, n}=d_{m, n}+c_{m}$ and $d_{m, n+1}=d_{m, n}-c_{n}^{\prime}$, as a consequence of the formulae $\left[E, V_{X}(c)\right]=c V_{X}(c)$ and $\left[E, V_{Y}\left(c^{\prime}\right)\right]=-c^{\prime} V_{Y}\left(c^{\prime}\right)$; cf. [Tsu09b, Lemma 2.4]. Hence the balancing condition

$$
d_{m, n}+d_{m+1, n+1}=d_{m, n+1}+d_{m+1, n}
$$

is fulfilled. We call the above sequence of homogeneous solutions of the UC hierarchy a homogeneous $\tau$-sequence. Obviously, any functional equation in Sect. 2 still remains valid for the homogeneous $\tau$-sequence $\left\{\tau_{m, n}\right\}$; we may call also $V_{X}(c)$ and $V_{Y}\left(c^{\prime}\right)$ vertex operators.

Example 3.1. If we take $c=c^{\prime}=n$ to be an integer and each $\gamma$ and $\gamma^{\prime}$ a positively oriented small circle around the origin $z=0$, then $V_{X}(n)=2 \pi \sqrt{-1} X_{n}^{+}$and $V_{Y}(n)=2 \pi \sqrt{-1} Y_{n}^{+}$according to (1.2). Recall now that these operators play roles of raising operators for the universal characters; namely,

$$
S_{[\lambda, \mu]}(\boldsymbol{x}, \boldsymbol{y})=X_{\lambda_{1}}^{+} \ldots X_{\lambda_{\ell}}^{+} Y_{\mu_{1}}^{+} \ldots Y_{\mu_{\ell^{\prime}}}^{+}, 1
$$

for any pair of partitions $\lambda=\left(\lambda_{1}, \lambda_{2}, \ldots, \lambda_{\ell}\right)$ and $\mu=\left(\mu_{1}, \mu_{2}, \ldots, \mu_{\ell^{\prime}}\right)$; see [Tsu04, Theorem 1.2]. Starting from a trivial solution $\tau(\boldsymbol{x}, \boldsymbol{y})=S_{[\emptyset, \emptyset]}(\boldsymbol{x}, \boldsymbol{y}) \equiv 1$ of the UC hierarchy, we thus obtain a homogeneous $\tau$-sequence expressed in the universal characters by successive application of $X_{n}^{+}$ and $Y_{n}^{n}$.

Next we consider the Weyl group symmetry of the homogeneous $\tau$-sequence. Fix a positive integer $k$. Let us look at $m=k$ cites in the $(m, n)$-lattice and interchange the $(k-1)$ th and $k$ th operations of the vertex operators $V_{X}$ in view of the fermionic relation $V_{X}(a) V_{X}(b)+V_{X}(b-1) V_{X}(a+$ $1)=0$. To be more precise, we transform the original sequence

$$
\cdots \stackrel{V_{X}\left(c_{k-2}\right)}{\longrightarrow} \tau_{k-1, n} \stackrel{V_{X}\left(c_{k-1}\right)}{\longrightarrow} \tau_{k, n} \stackrel{V_{X}\left(c_{k}\right)}{\longrightarrow} \tau_{k+1, n} \stackrel{V_{X}\left(c_{k+1}\right)}{\longrightarrow} \cdots
$$

into a new one

$$
\ldots \stackrel{V_{X}\left(c_{k-2}\right)}{\longrightarrow} \tau_{k-1, n} \stackrel{V_{X}\left(c_{k}+1\right)}{\longrightarrow} \hat{\tau}_{k, n} \stackrel{V_{X}\left(c_{k-1}-1\right)}{\longrightarrow} \tau_{k+1, n} \stackrel{V_{X}\left(c_{k+1}\right)}{\longrightarrow} \cdots
$$

that is identical with the original one except $\tau_{k, n}$ is replaced by

$$
\hat{\tau}_{k, n}=V_{X}\left(c_{k}+1\right) \tau_{k-1, n} .
$$

Besides, the degree of $\hat{\tau}_{k, n}$ reads

$$
\hat{d}_{k, n}=d_{k-1, n}+c_{k}+1=d_{k-1, n}-d_{k, n}+d_{k+1, n}+1 .
$$

We refer to the above permutation of vertex operators as $r_{k}$. Put

$$
\alpha_{k}=\hat{d}_{k, n}-d_{k, n}=d_{k-1, n}-2 d_{k, n}+d_{k+1, n}+1,
$$

which is a quantity that does not depend on $n$. The operation $r_{k}$ induces the transformation

$$
r_{k}\left(\alpha_{k}\right)=-\alpha_{k}, \quad r_{k}\left(\alpha_{k \pm 1}\right)=\alpha_{k \pm 1}+\alpha_{k}, \quad \text { and } \quad r_{k}\left(\alpha_{\ell}\right)=\alpha_{\ell} \quad(\ell \neq k, k \pm 1) .
$$


Therefore $\alpha_{k}$ can be regarded as a root variable of the Weyl group of type $A$, and $\left\langle r_{k}\right\rangle$ indeed fulfills its fundamental relations

$$
r_{k}^{2}=1, \quad r_{k} r_{k \pm 1} r_{k}=r_{k \pm 1} r_{k} r_{k \pm 1}, \quad \text { and } \quad r_{k} r_{\ell}=r_{\ell} r_{k} \quad(\ell \neq k, k \pm 1) .
$$

Along the same lines we can derive from a permutation of operators $V_{Y}$ another action of the Weyl group of type $A$, which commutes with the previous one. As demonstrated in Sect 8, this kind of Weyl group actions gives rise to a group of birational canonical transformations of the Hamiltonian system $\mathcal{H}_{L, N}$.

We conclude this section with some formulae that will be employed later.

Lemma 3.2. It holds that

$$
\begin{aligned}
& \tau_{k-1, n} \otimes \tau_{k+1, n}-\sum_{i+j=-1} X_{i}^{-} \hat{\tau}_{k, n} \otimes X_{j}^{+} \tau_{k, n}=\sum_{i+j=-1} Y_{i}^{-} \hat{\tau}_{k, n} \otimes Y_{j}^{+} \tau_{k, n}=0, \\
& \tau_{k-1, n+1} \otimes \tau_{k+1, n}-\sum_{i+j=-1} X_{i}^{-} \hat{\tau}_{k, n+1} \otimes X_{j}^{+} \tau_{k, n}=\hat{\tau}_{k, n} \otimes \tau_{k, n+1}-\sum_{i+j=0} Y_{i}^{-} \hat{\tau}_{k, n+1} \otimes Y_{j}^{+} \tau_{k, n}=0 .
\end{aligned}
$$

Proof. First we have

$$
\sum_{i+j=-2} X_{i}^{-} \tau_{k-1, n} \otimes X_{j}^{+} \tau_{k, n}=\sum_{i+j=-1} Y_{i}^{-} \tau_{k-1, n} \otimes Y_{j}^{+} \tau_{k, n}=0
$$

which is equivalent to (2.2) with $m=1$ and $n=0$. Applying $V_{X}\left(c_{k}+2\right) \otimes 1$ and $V_{X}\left(c_{k}+1\right) \otimes 1$ respectively to the first and second equalities in (3.4) leads to (3.2). We deduce (3.3) from (3.2) by applying $V_{Y}\left(c_{n}^{\prime}\right) \otimes 1$.

Lemma 3.3. The following difference (and differential) equations hold:

$$
\begin{aligned}
& D_{\delta_{t}} \hat{\tau}_{k, n}(\boldsymbol{x}, \boldsymbol{y}) \cdot \tau_{k, n}(\boldsymbol{x}, \boldsymbol{y})-t \tau_{k-1, n}\left(\boldsymbol{x}-[t], \boldsymbol{y}-\left[t^{-1}\right]\right) \tau_{k+1, n}\left(\boldsymbol{x}+[t], \boldsymbol{y}+\left[t^{-1}\right]\right)=0, \\
& t \tau_{k-1, n+1}\left(\boldsymbol{x}-[t], \boldsymbol{y}-\left[t^{-1}\right]\right) \tau_{k+1, n}(\boldsymbol{x}, \boldsymbol{y}) \\
& \quad-\hat{\tau}_{k, n+1}(\boldsymbol{x}, \boldsymbol{y}) \tau_{k, n}\left(\boldsymbol{x}-[t], \boldsymbol{y}-\left[t^{-1}\right]\right)+\hat{\tau}_{k, n}\left(\boldsymbol{x}-[t], \boldsymbol{y}-\left[t^{-1}\right]\right) \tau_{k, n+1}(\boldsymbol{x}, \boldsymbol{y})=0 .
\end{aligned}
$$

Proof. The verification can be done along the same argument as Proposition 2.2. First we shall regard the symbol $f \otimes g$ as a product of two functions $f\left(\boldsymbol{x}^{\prime}, \boldsymbol{y}^{\prime}\right) g(\boldsymbol{x}, \boldsymbol{y})$ in distinct indeterminates $\left(\boldsymbol{x}^{\prime}, \boldsymbol{y}^{\prime}\right)$ and $(\boldsymbol{x}, \boldsymbol{y})$. Taking the variables in (3.2) as $\boldsymbol{x}-\boldsymbol{x}^{\prime}=2[t]$ and $\boldsymbol{y}-\boldsymbol{y}^{\prime}=2\left[t^{-1}\right]$ thus leads to (3.5). Similarly, we deduce (3.6) from (3.3) with $\boldsymbol{x}-\boldsymbol{x}^{\prime}=[t]$ and $\boldsymbol{y}-\boldsymbol{y}^{\prime}=\left[t^{-1}\right]$.

\section{Similarity reduction of UC hierarchy}

In this section we consider a reduction of the UC hierarchy by requiring certain homogeneity and periodicity. As a result we derive a finite-dimensional integrable system of partial differential equations, denoted by $\mathcal{G}_{L, N}$, which provides an extension of both the Garnier system and the sixth Painlevé equation $P_{\mathrm{VI}}$.

Fix integers $L \geq 2$ and $N \geq 1$. Let $\tau_{m, n}=\tau_{m, n}(\boldsymbol{x}, \boldsymbol{y})$ be a sequence of the solutions satisfying (2.15)-(2.17) in Proposition 2.4. Suppose that $\tau_{m, n}$ are homogeneous of degree $d_{m, n} \in \mathbb{C}$, i.e.,

$$
E \tau_{m, n}=d_{m, n} \tau_{m, n} \quad \text { with } \quad E=\sum_{n=1}^{\infty}\left(n x_{n} \frac{\partial}{\partial x_{n}}-n y_{n} \frac{\partial}{\partial y_{n}}\right),
$$


and fullfil the periodic condition: $\tau_{m+L, n}=\tau_{m, n+L}=\tau_{m, n}$ (up to multiplication by constants). Remark here that the relation $d_{m, n}+d_{m+1, n+1}=d_{m, n+1}+d_{m+1, n}$ necessarily holds; cf. Sect. 3. Let us replace the independent variables $x_{n}$ and $y_{n}$ respectively with the $n$th and $(-n)$ th power sum of new ones $\boldsymbol{t}=\left(t_{0}, t_{1}, \ldots, t_{N}\right)$ as

$$
x_{n}=\frac{1}{n} \sum_{i=0}^{N} \theta_{i} t_{i}^{n} \quad \text { and } \quad y_{n}=\frac{1}{n} \sum_{i=0}^{N} \theta_{i} t_{i}^{-n} .
$$

Consequently we have

$$
\begin{aligned}
t_{i} \frac{\partial}{\partial t_{i}} & =t_{i} \sum_{n=1}^{\infty}\left(\frac{\partial x_{n}}{\partial t_{i}} \frac{\partial}{\partial x_{n}}+\frac{\partial y_{n}}{\partial t_{i}} \frac{\partial}{\partial y_{n}}\right)=\theta_{i} \sum_{n=1}^{\infty}\left(t_{i}^{n} \frac{\partial}{\partial x_{n}}-t_{i}^{-n} \frac{\partial}{\partial y_{n}}\right)=\theta_{i} \delta_{t_{i}}, \\
E & =\sum_{i=0}^{N} t_{i} \frac{\partial}{\partial t_{i}}=\sum_{i=0}^{N} \theta_{i} \delta_{t_{i}} .
\end{aligned}
$$

In view of the homogeneity, no generality is lost by taking $t_{0}=1$. Set $\sigma_{m, n}(\boldsymbol{\theta}, \boldsymbol{t})=\tau_{m, n}(\boldsymbol{x}, \boldsymbol{y})$ under the above conditions. For notational simplicity we shall use the abbreviation $\sigma_{m, n}\left(\theta_{i} \pm 1\right)$ to mean that among the constant parameters $\boldsymbol{\theta}=\left(\theta_{0}, \theta_{1}, \ldots, \theta_{N}\right)$ only the indicated one $\theta_{i}$ is shifted by \pm 1 while all the others are unchanged. Then we have the

Proposition 4.1. The functions $\sigma_{m, n}=\sigma_{m, n}(\boldsymbol{\theta}, \boldsymbol{t})$ satisfy the bilinear equations

$$
\begin{aligned}
& \left(t_{i}-t_{j}\right) \sigma_{m, n} \sigma_{m+1, n+1}\left(\theta_{i}+1, \theta_{j}+1\right) \\
& \quad \quad=t_{i} \sigma_{m+1, n}\left(\theta_{i}+1\right) \sigma_{m, n+1}\left(\theta_{j}+1\right)-t_{j} \sigma_{m+1, n}\left(\theta_{j}+1\right) \sigma_{m, n+1}\left(\theta_{i}+1\right), \\
& \left(t_{i} D_{i}+\theta_{i}\right) \sigma_{m+1, n} \cdot \sigma_{m, n+1}=\theta_{i} \sigma_{m, n}\left(\theta_{i}-1\right) \sigma_{m+1, n+1}\left(\theta_{i}+1\right), \\
& \left(\left(t_{j}-t_{i}\right) D_{i}+\theta_{i}\right) \sigma_{m, n}\left(\theta_{j}-1\right) \cdot \sigma_{m+1, n}=\theta_{i} \sigma_{m, n}\left(\theta_{i}-1\right) \sigma_{m+1, n}\left(\theta_{i}+1, \theta_{j}-1\right),
\end{aligned}
$$

together with the homogeneity constraint

$$
\sum_{i=0}^{N} t_{i} \frac{\partial \sigma_{m, n}}{\partial t_{i}}=d_{m, n} \sigma_{m, n} .
$$

Here $D_{i}$ denotes the Hirota differential with respect to $\partial / \partial t_{i}$.

Proof. It is immediate to obtain (4.4a) from (2.15) with $(t, s)=\left(t_{i}, t_{j}\right)$. Using (4.2) we verify (4.4b) and (4.4c) from (2.16) and (2.17), respectively.

Next we shall write down nonlinear differential equations for appropriately chosen dependent variables. Let us introduce the functions $f_{m, n}^{(i)}=f_{m, n}^{(i)}(\boldsymbol{\theta}, \boldsymbol{t})$ and $g_{m, n}^{(i)}=g_{m, n}^{(i)}(\boldsymbol{\theta}, \boldsymbol{t})$ defined by

$$
\begin{aligned}
f_{m, n}^{(i)} & =\frac{\sigma_{m, n-1}\left(\theta_{i}+1\right) \sigma_{m-1, n-1}}{\sigma_{m-1, n}\left(\theta_{i}+1\right) \sigma_{m, n-2}} \\
g_{m, n}^{(i)} & =\frac{t_{i} D_{i} \sigma_{m, n-1} \cdot \sigma_{m-1, n}}{\sigma_{m, n-1} \sigma_{m-1, n}}+\theta_{i}=\theta_{i} \frac{\sigma_{m-1, n-1}\left(\theta_{i}-1\right) \sigma_{m, n}\left(\theta_{i}+1\right)}{\sigma_{m, n-1} \sigma_{m-1, n}}
\end{aligned}
$$


for $i=0,1, \ldots, N$. Note that the second equality in (4.6) is a consequence of (4.4b). We have the conservation law

$$
\prod_{j=1}^{L} f_{m+j, n-j}^{(i)}=1 \quad \text { and } \quad \sum_{j=1}^{L} g_{m+j, n-j}^{(i)}=L \theta_{i} .
$$

In addition we prepare auxiliary variables $U_{m, n}^{(i, j)}$ and $V_{m, n}^{(i, j)}(i \neq j)$ defined by

$$
\begin{aligned}
U_{m, n}^{(i, j)} & =\frac{\theta_{i} t_{j}}{t_{i}-t_{j}} \frac{\sigma_{m, n-1}\left(\theta_{i}-1, \theta_{j}+1\right) \sigma_{m, n}\left(\theta_{i}+1\right)}{\sigma_{m, n-1} \sigma_{m, n}\left(\theta_{j}+1\right)}, \\
V_{m, n}^{(i, j)} & =\frac{\theta_{i} t_{i}}{t_{i}-t_{j}} \frac{\sigma_{m-1, n}\left(\theta_{i}-1, \theta_{j}+1\right) \sigma_{m, n}\left(\theta_{i}+1\right)}{\sigma_{m-1, n} \sigma_{m, n}\left(\theta_{j}+1\right)} .
\end{aligned}
$$

Then we have the following relations among the dependent variables.

Lemma 4.2. For $i \neq j$, it holds that

$$
\begin{aligned}
V_{m, n}^{(i, j)}-U_{m, n}^{(i, j)} & =g_{m, n}^{(i)}, \\
\frac{U_{m-1, n}^{(i, j)}}{V_{m, n-1}^{(i, j)}} & =\frac{t_{j} f_{m, n}^{(j)}}{t_{i} f_{m, n}^{(i)}}, \\
V_{m, n-1}^{(i, j)}-U_{m-1, n}^{(i, j)} & =g_{m, n}^{(i)}\left(\theta_{j}+1\right), \\
\frac{U_{m, n}^{(i, j)}}{V_{m, n}^{(i, j)}} & =\frac{t_{j} f_{m, n}^{(j)}\left(\theta_{i}-1\right)}{t_{i} f_{m, n}^{(i)}\left(\theta_{i}-1\right)} .
\end{aligned}
$$

Proof. Clearly (4.9) and (4.11) are direct consequences of the definition of $f_{m, n}^{(i)},(4.5)$. We obtain (4.8) and (4.10) from the bilinear equation (4.4a).

Solving the linear equations (4.8) and (4.9) for $U_{m, n}^{(i, j)}$ and $V_{m, n}^{(i, j)}$ with the aid of the $(L, L)$ periodicity, we conclude that

$$
\begin{aligned}
U_{m, n}^{(i, j)} & =\frac{1}{\left(\frac{t_{i}}{t_{j}}\right)^{L}-1} \sum_{b=1}^{L} g_{m-b+1, n+b-1}^{(i)} \prod_{a=1}^{b-1} \frac{t_{i} f_{m-a+1, n+a}^{(i)}}{t_{j} f_{m-a+1, n+a}^{(j)}}, \\
V_{m, n}^{(i, j)} & =\frac{1}{\left(\frac{t_{i}}{t_{j}}\right)^{L}-1} \sum_{b=1}^{L} g_{m-b, n+b}^{(i)} \prod_{a=0}^{b-1} \frac{t_{i} f_{m-a, n+a+1}^{(i)}}{t_{j} f_{m-a, n+a+1}^{(j)}}
\end{aligned}
$$

In fact $U_{m, n}^{(i, j)}$ and $V_{m, n}^{(i, j)}$ can be expressed as polynomials in $f_{m, n}^{(i)}$ and $g_{m, n}^{(i)}$ via (4.7). 
Theorem 4.3. The functions $f_{m, n}^{(i)}$ and $g_{m, n}^{(i)}$ satisfy the system of nonlinear differential equations

$$
\begin{aligned}
t_{i} \frac{\partial f_{m, n}^{(i)}}{\partial t_{i}} & =\left(\kappa_{m, n}-g_{m, n-1}^{(i)}+\sum_{j \neq i}\left(U_{m-1, n}^{(j, i)}-V_{m, n-1}^{(j, i)}\right)\right) f_{m, n}^{(i)}, \\
t_{j} \frac{\partial f_{m, n}^{(i)}}{\partial t_{j}} & =\left(-g_{m, n-1}^{(i)}-U_{m-1, n}^{(j, i)}+V_{m, n-1}^{(j, i)}\right) f_{m, n}^{(i)} \quad(i \neq j), \\
t_{i} \frac{\partial g_{m, n}^{(i)}}{\partial t_{i}} & =-\sum_{j \neq i}\left(U_{m, n}^{(i, j)} g_{m, n}^{(j)}+V_{m, n}^{(j, i)} g_{m, n}^{(i)}\right), \\
t_{j} \frac{\partial g_{m, n}^{(i)}}{\partial t_{j}} & =U_{m, n}^{(i, j)} g_{m, n}^{(j)}+V_{m, n}^{(j, i)} g_{m, n}^{(i)} \quad(i \neq j),
\end{aligned}
$$

where

$$
\kappa_{m, n}=d_{m, n-1}-d_{m-1, n}+\sum_{i=0}^{N} \theta_{i}=\sum_{i=0}^{N} g_{m, n}^{(i)} \in \mathbb{C}
$$

are constant parameters.

For each $(m, n)$ fixed the system (4.12) is closed with respect to the $2 L N$-tuple of dependent variables $g_{m+j, n-j}^{(i)}$ and $f_{m+j, n-j+1}^{(i)} / f_{m+j, n-j+1}^{(0)}$, where $i=1,2, \ldots, N$ and $j \in \mathbb{Z} / L \mathbb{Z}$. Moreover, it possesses the $2 N$ conserved quantities; recall (4.7). Accordingly the dimension of the phase space is essentially $2 N(L-1)$. If $L=2$ then it is in fact equivalent to the Garnier system in $N$ variables, whose phase space is $2 N$-dimensional; see also the appendix. Let $\mathcal{G}_{L, N}$ denote the nonlinear system (4.12). As shown in Sect. 7, the system $\mathcal{G}_{L, N}$ can be transformed into a canonical Hamiltonian system with polynomial Hamiltonian functions.

Proof of Theorem 4.3. We shall demonstrate only (4.12a) here because the others can be done in quite a similar manner. By virtue of the homogeneity $(4.4 \mathrm{~d})$ we see that

$$
\sum_{i=0}^{N}\left(g_{m, n}^{(i)}-\theta_{i}\right)=\sum_{i=0}^{N} \frac{t_{i} D_{i} \sigma_{m . n-1} \cdot \sigma_{m-1, n}}{\sigma_{m . n-1} \sigma_{m-1, n}}=d_{m, n-1}-d_{m-1, n} .
$$

Therefore (4.13) certainly holds. By combining (4.10) with (4.13) we have also

$$
\begin{aligned}
g_{m, n}^{(i)}\left(\theta_{i}+1\right) & =\kappa_{m, n}+1-\sum_{j \neq i} g_{m, n}^{(j)}\left(\theta_{i}+1\right) \\
& =\kappa_{m, n}+1+\sum_{j \neq i}\left(U_{m-1, n}^{(j, i)}-V_{m, n-1}^{(j, i)}\right) .
\end{aligned}
$$

Taking the logarithmic derivative of $f_{m, n}^{(i)}$ shows that

$$
\begin{aligned}
\frac{t_{i}}{f_{m, n}^{(i)}} \frac{\partial f_{m, n}^{(i)}}{\partial t_{i}} & =\frac{t_{i} D_{i} \sigma_{m, n-1}\left(\theta_{i}+1\right) \cdot \sigma_{m-1, n}\left(\theta_{i}+1\right)}{\sigma_{m, n-1}\left(\theta_{i}+1\right) \sigma_{m-1, n}\left(\theta_{i}+1\right)}-\frac{t_{i} D_{i} \sigma_{m, n-2} \cdot \sigma_{m-1, n-1}}{\sigma_{m, n-2} \sigma_{m-1, n-1}} \\
& =g_{m, n}^{(i)}\left(\theta_{i}+1\right)-g_{m, n-1}^{(i)}-1, \quad \operatorname{using}(4.6), \\
& =\kappa_{m, n}-g_{m, n-1}^{(i)}+\sum_{j \neq i}\left(U_{m-1, n}^{(j, i)}-V_{m, n-1}^{(j, i)}\right), \quad \text { using (4.14). }
\end{aligned}
$$

We have verified (4.12a) as desired. 
Remark 4.4 (Toda equation). We shall derive a differential-difference equation of Toda-type for $\sigma_{m, n}$, associated with the shift $\left(\theta_{i}, \theta_{j}\right) \mapsto\left(\theta_{i}+1, \theta_{j}-1\right)$ of parameters. First we differentiate with respect to $s$ the equation (2.17) after shifting the variables $(\boldsymbol{x}, \boldsymbol{y})$ to $\left(\boldsymbol{x}+[s] / 2, \boldsymbol{y}+\left[s^{-1}\right] / 2\right)$. We thus find that

$$
\begin{aligned}
& \left((s-t) D_{\delta_{t}} D_{\delta_{s}}-2 s D_{\delta_{t}}+t D_{\delta_{s}}\right) \tau_{m, n}\left(\boldsymbol{x}-\frac{[s]}{2}, \boldsymbol{y}-\frac{\left[s^{-1}\right]}{2}\right) \cdot \tau_{m+1, n}\left(\boldsymbol{x}+\frac{[s]}{2}, \boldsymbol{y}+\frac{\left[s^{-1}\right]}{2}\right) \\
& \quad+t D_{\delta_{s}} \tau_{m, n}\left(\boldsymbol{x}-[t]+\frac{[s]}{2}, \boldsymbol{y}-\left[t^{-1}\right]+\frac{\left[s^{-1}\right]}{2}\right) \cdot \tau_{m+1, n}\left(\boldsymbol{x}+[t]-\frac{[s]}{2}, \boldsymbol{y}+\left[t^{-1}\right]-\frac{\left[s^{-1}\right]}{2}\right)=0 .
\end{aligned}
$$

Substitution of $(4.1)$ and $(t, s)=\left(t_{i}, t_{j}\right)$ leads to

$$
\begin{gathered}
\left(\left(t_{j}-t_{i}\right) D_{i} D_{j}-\left(2 \theta_{j}+1\right) D_{i}+\theta_{i} D_{j}\right) \sigma_{m, n} \cdot \sigma_{m+1, n}\left(\theta_{j}+1\right) \\
+\theta_{i} D_{j} \sigma_{m, n}\left(\theta_{i}-1, \theta_{j}+1\right) \cdot \sigma_{m+1, n}\left(\theta_{i}+1\right)=0
\end{gathered}
$$

Hence, with the aid of $(4.4 \mathrm{c})$, we verify that

$$
\begin{aligned}
\left(t_{i}-t_{j}\right)^{2} & \frac{D_{i} D_{j} \sigma_{m, n} \cdot \sigma_{m+1, n}\left(\theta_{j}+1\right)}{\sigma_{m, n} \sigma_{m+1, n}\left(\theta_{j}+1\right)} \\
=- & \theta_{i}\left(2 \theta_{j}+1\right)+\theta_{i} \theta_{j} \frac{\sigma_{m, n}\left(\theta_{i}+1, \theta_{j}-1\right) \sigma_{m, n}\left(\theta_{i}-1, \theta_{j}+1\right)}{\sigma_{m, n}{ }^{2}} \\
& +\theta_{i}\left(\theta_{j}+1\right) \frac{\sigma_{m+1, n}\left(\theta_{i}+1\right) \sigma_{m+1, n}\left(\theta_{i}-1, \theta_{j}+2\right)}{\sigma_{m+1, n}\left(\theta_{j}+1\right)^{2}} \\
& +\left(t_{i}-t_{j}\right)^{2}\left(\frac{D_{i} \sigma_{m, n} \cdot \sigma_{m+1, n}\left(\theta_{j}+1\right)}{\sigma_{m, n} \sigma_{m+1, n}\left(\theta_{j}+1\right)}\right)\left(\frac{D_{j} \sigma_{m, n} \cdot \sigma_{m+1, n}\left(\theta_{j}+1\right)}{\sigma_{m, n} \sigma_{m+1, n}\left(\theta_{j}+1\right)}\right) .
\end{aligned}
$$

Next we express $(4.4 c)$ in the form

$$
\left(t_{i}-t_{j}\right) \frac{D_{i} \sigma_{m, n} \cdot \sigma_{m+1, n}\left(\theta_{j}+1\right)}{\sigma_{m, n} \sigma_{m+1, n}\left(\theta_{j}+1\right)}=\theta_{i}-\theta_{i} \frac{\sigma_{m, n}\left(\theta_{i}-1, \theta_{j}+1\right) \sigma_{m+1, n}\left(\theta_{i}+1\right)}{\sigma_{m, n} \sigma_{m+1, n}\left(\theta_{j}+1\right)} .
$$

By differentiating this with respect to $t_{j}$, we have

$$
\begin{aligned}
\left(t_{i}-t_{j}\right)^{2} \frac{\partial}{\partial t_{j}}\left(\frac{D_{i} \sigma_{m, n} \cdot \sigma_{m+1, n}\left(\theta_{j}+1\right)}{\sigma_{m, n} \sigma_{m+1, n}\left(\theta_{j}+1\right)}\right) & \\
=\theta_{i} & +\theta_{i} \theta_{j} \frac{\sigma_{m, n}\left(\theta_{i}+1, \theta_{j}-1\right) \sigma_{m, n}\left(\theta_{i}-1, \theta_{j}+1\right)}{\sigma_{m, n}^{2}} \\
& \quad \theta_{i}\left(\theta_{j}+1\right) \frac{\sigma_{m+1, n}\left(\theta_{i}+1\right) \sigma_{m+1, n}\left(\theta_{i}-1, \theta_{j}+2\right)}{\sigma_{m+1, n}\left(\theta_{j}+1\right)^{2}} .
\end{aligned}
$$

Finally, combining (4.15) and (4.16), we arive at the Toda equation:

$$
\left(t_{i}-t_{j}\right)^{2} \frac{D_{i} D_{j} \sigma_{m, n} \cdot \sigma_{m, n}}{\sigma_{m, n}{ }^{2}}=-2 \theta_{i} \theta_{j}+2 \theta_{i} \theta_{j} \frac{\sigma_{m, n}\left(\theta_{i}+1, \theta_{j}-1\right) \sigma_{m, n}\left(\theta_{i}-1, \theta_{j}+1\right)}{\sigma_{m, n}{ }^{2}} .
$$

Note that (4.17) is still valid without requiring the homogeneity and periodicity. Such a differentialdifference equation of Toda-type has previously been studied for the case of $P_{\mathrm{VI}}$, i.e., $(L, N)=(2,1)$ by Okamoto [Oka87]; and for the case of the Garnier systems, i.e., $L=2$ and general $N \geq 1$, refer to [Tsu06]. 


\section{Particular solutions expressed in terms of the universal character}

As described in Sect. 4, the system $\mathcal{G}_{L, N}$ is a similarity reduction of the UC hierarchy. Since the universal characters $S_{[\lambda, \mu]}=S_{[\lambda, \mu]}(\boldsymbol{x}, \boldsymbol{y})$ are homogeneous solutions of the UC hierarchy, they survive through the reduction procedure; recall Example 3.1. Therefore we can immediately construct a solution of $\mathcal{G}_{L, N}$ expressed in terms of the universal character.

First we recall some terminology. A subset $\mathbf{m} \subset \mathbb{Z}$ is said to be a Maya diagram if $i \in \mathbf{m}$ (for $i \ll 0$ ) and $i \notin \mathbf{m}$ (for $i \gg 0$ ). Each Maya diagram $\mathbf{m}=\left\{\ldots, m_{3}, m_{2}, m_{1}\right\}$ corresponds to a partition $\lambda=\left(\lambda_{1}, \lambda_{2}, \ldots\right)$ via $m_{i}-m_{i+1}=\lambda_{i}-\lambda_{i+1}+1$. We can associate with a sequence of integers $\boldsymbol{v}=\left(v_{1}, v_{2}, \ldots, v_{L}\right) \in \mathbb{Z}^{L}$ a Maya diagram

$$
\mathbf{m}(v)=\left(L \mathbb{Z}_{<v_{1}}+1\right) \cup\left(L \mathbb{Z}_{<v_{2}}+2\right) \cup \cdots \cup\left(L \mathbb{Z}_{<v_{L}}+L\right) ;
$$

let $\lambda(\boldsymbol{v})$ denote its corresponding partition. Note that $\lambda(\boldsymbol{v}+\mathbf{1})=\lambda(\boldsymbol{v})$ where $\mathbf{1}=(\overbrace{1,1, \ldots, 1}^{L})$. We call a partition of the form $\lambda(v)$ an $L$-core partition. A partition $\lambda$ is $L$-core if and only if $\lambda$ has no hook with length of a multiple of $L$; see [Nou04, Proposition 7.13]. For example, if $L=2$ and $v=(0, n)(n>0)$ then the result is a staircase partition $\lambda(v)=(n, n-1, \ldots, 2,1)$, thereby two-core.

There is a cyclic chain of the universal characters attached to $L$-core partitions that is connected by the action of vertex operators; see [Tsu05a, Lemma 2.2].

Lemma 5.1. It holds that

$$
X_{L v_{m}-|v|}^{+} S_{[\lambda(\boldsymbol{v}(m-1)), \mu]}= \pm S_{[\lambda(\boldsymbol{v}(m)), \mu]}
$$

for arbitrary $\boldsymbol{v}=\left(v_{1}, v_{2}, \ldots, v_{L}\right) \in \mathbb{Z}^{L}$ and partition $\mu$. Here $\boldsymbol{v}(m)=v+(\overbrace{1, \ldots, 1}^{m}, \overbrace{0, \ldots, 0}^{L-m})$ and $|v|=v_{1}+v_{2}+\cdots+v_{L}$. A similar formula holds for the operators $Y_{n}^{+}$also.

Hence we are led to the following expression of rational solutions of $\mathcal{G}_{L, N}$ in terms of the universal character attached to a pair of $L$-core partitions.

Theorem 5.2. Let $\boldsymbol{v}, \boldsymbol{v}^{\prime} \in \mathbb{Z}^{L}$ be arbitrary sequences of integers. Define

$$
\sigma_{m, n}(\boldsymbol{\theta}, \boldsymbol{t})=S_{\left[\lambda(\boldsymbol{v}(m)), \lambda\left(\boldsymbol{v}^{\prime}(n)\right)\right]}(\boldsymbol{x}, \boldsymbol{y})
$$

under the substitution

$$
x_{n}=\frac{1}{n} \sum_{i=0}^{N} \theta_{i} t_{i}^{n} \quad \text { and } \quad y_{n}=\frac{1}{n} \sum_{i=0}^{N} \theta_{i} t_{i}^{-n} .
$$

Then the functions $\sigma_{m, n}$ satisfy the bilinear equations (4.4a)-(4.4c) and the homogeneity (4.4d), where $d_{m, n}-d_{m-1, n}=L v_{m}-|v|$ and $d_{m, n}-d_{m, n-1}=-L v_{n}^{\prime}+\left|v^{\prime}\right|$. Consequently the functions $f_{m, n}^{(i)}$ and $g_{m, n}^{(i)}$ defined by (4.5) and (4.6) give a rational solution of the system $\mathcal{G}_{L, N}$, (4.12), with the parameters $\kappa_{m, n}=L\left(v_{m}+v_{n}^{\prime}\right)-|\boldsymbol{v}|-\left|\boldsymbol{v}^{\prime}\right|+\sum_{i=0}^{N} \theta_{i}$.

\section{Lax formalism}

In this section we derive from the UC hierarchy the auxiliary linear problem whose compatibility condition amounts to the nonlinear system $\mathcal{G}_{L, N}$ (Lax formalism). It is seen that $\mathcal{G}_{L, N}$ describes the 
monodromy preserving deformations of a Fuchsian system of linear differential equations with a certain spectral type.

We introduce the wave function

$$
\psi_{m, n}(\boldsymbol{x}, \boldsymbol{y}, k)=\frac{\tau_{m, n-1}\left(\boldsymbol{x}-\left[k^{-1}\right], \boldsymbol{y}-[k]\right)}{\tau_{m, n}(\boldsymbol{x}, \boldsymbol{y})} e^{\xi(\boldsymbol{x}, k)}
$$

which is a function in $(\boldsymbol{x}, \boldsymbol{y})=\left(x_{1}, x_{2}, \ldots, y_{1}, y_{2}, \ldots\right)$ equipped with an additional parameter $k$ (the spectral variable $)$. Define $\phi_{m, n}(\boldsymbol{\theta}, \boldsymbol{t}, k)=\psi_{m, n}(\boldsymbol{x}, \boldsymbol{y}, k)$ under the change of variables (4.1). We then have the

Proposition 6.1. The wave functions $\phi_{m, n}=\phi_{m, n}(\boldsymbol{\theta}, \boldsymbol{t}, k)$ satisfy the linear equations

$$
\begin{aligned}
\phi_{m, n} & =\frac{1}{f_{m+1, n+1}^{(i)}} \phi_{m, n+1}\left(\theta_{i}+1\right)-t_{i} k \phi_{m+1, n}\left(\theta_{i}+1\right), \\
t_{i} \frac{\partial}{\partial t_{i}} \phi_{m, n} & =\left(g_{m+1, n}^{(i)}-\theta_{i}\right) \phi_{m, n}+t_{i} k g_{m+1, n}^{(i)} \phi_{m+1, n}\left(\theta_{i}+1\right), \\
\left(k \frac{\partial}{\partial k}-\sum_{i=0}^{N} t_{i} \frac{\partial}{\partial t_{i}}\right) \phi_{m, n} & =\left(d_{m, n}-d_{m, n-1}\right) \phi_{m, n} .
\end{aligned}
$$

Proof. To begin with, we recall the definition of variables $f_{m, n}^{(i)}$ and $g_{m, n}^{(i)}$; see (4.5) and (4.6). Substitution of $(t, s)=\left(t_{i}, 1 / k\right)$ in (2.15) and (2.17) produces respectively (6.1) and (6.2), with the aid of (4.2). We deduce from the homogeneity condition $E \tau_{m, n}=d_{m, n} \tau_{m, n}$ that

$$
\left(E-k \frac{\partial}{\partial k}\right) \tau_{m, n}\left(\boldsymbol{x}-\left[k^{-1}\right], \boldsymbol{y}-[k]\right)=d_{m, n} \tau_{m, n}\left(\boldsymbol{x}-\left[k^{-1}\right], \boldsymbol{y}-[k]\right) .
$$

On the other hand, we have $(E-k \partial / \partial k) e^{\xi(x, k)}=0$. Hence we are led to the formula

$$
\left(E-k \frac{\partial}{\partial k}\right) \psi_{m, n}=\left(d_{m, n-1}-d_{m, n}\right) \psi_{m, n}
$$

which implies (6.3) via (4.3). The proof is now complete.

Because of the $(L, L)$-periodicity, the linear equations (6.1) can be solved for $\phi_{m, n}\left(\theta_{i}+1\right)$; thus,

$$
\phi_{m, n}\left(\theta_{i}+1\right)=\frac{1}{1-\left(t_{i} k\right)^{L}} \sum_{b=1}^{L}\left(t_{i} k\right)^{b-1}\left(\prod_{a=1}^{b} f_{m+a, n-a+1}^{(i)}\right) \phi_{m+b-1, n-b} .
$$

If we eliminate $\phi_{m+1, n}\left(\theta_{i}+1\right)$ from (6.2) by using the above formula, then we have

$$
t_{i} \frac{\partial}{\partial t_{i}} \phi_{m, n}=\left(g_{m+1, n}^{(i)}-\theta_{i}\right) \phi_{m, n}+\frac{g_{m+1, n}^{(i)}}{1-\left(t_{i} k\right)^{L}} \sum_{b=1}^{L}\left(t_{i} k\right)^{b}\left(\prod_{a=1}^{b} f_{m+a+1, n-a+1}^{(i)}\right) \phi_{m+b, n-b} .
$$

Notice that for each $m$ and $n$ fixed (6.4) is closed with respect to $\phi_{m+j, n-j}(j \in \mathbb{Z} / L \mathbb{Z})$. With this fact in mind, we shall write down the linear differential equations satisfied by the vector

$$
\Phi={ }^{\mathrm{T}}\left(\phi_{-1,0}, k \phi_{0,-1}, k^{2} \phi_{1,-2}, \ldots, k^{L-1} \phi_{L-2,-L+1}\right) .
$$


Consider the change of variables

$$
z=k^{L} \quad \text { and } \quad u_{i}=t_{i}^{-L}
$$

We can express (6.4) in the $L \times L$ matrix equation

$$
\frac{\partial}{\partial u_{i}} \Phi=B_{i} \Phi
$$

with

$$
\begin{aligned}
B_{i}= & \operatorname{diag}\left(\frac{\theta_{i}}{L u_{i}}-v_{n, n}^{(i)}\right)_{0 \leq n \leq L-1} \\
& +\frac{1}{z-u_{i}}\left(\begin{array}{cccc}
0 & v_{0,1}^{(i)} & \cdots & v_{0, L-1}^{(i)} \\
& 0 & \ddots & \vdots \\
& & \ddots & v_{L-2, L-1}^{(i)}
\end{array}\right)+\frac{z}{z-u_{i}}\left(\begin{array}{cccc}
v_{0,0}^{(i)} & & & O \\
v_{1,0}^{(i)} & v_{1,1}^{(i)} & & \\
\vdots & & \ddots & \\
v_{L-1,0}^{(i)} & v_{L-1,1}^{(i)} & \cdots & v_{L-1, L-1}^{(i)}
\end{array}\right),
\end{aligned}
$$

where

$$
v_{n, n+b}^{(i)}=\frac{g_{n,-n}^{(i)}}{L} \prod_{a=1}^{b} t_{i} f_{n+a,-n-a+1}^{(i)}
$$

for $0 \leq n \leq L-1$ and $1 \leq b \leq L$. Remark that the suffix of each variable should be suitably regarded as an element of $\mathbb{Z} / L \mathbb{Z}$.

Similarly, we obtain from (6.3) the linear differential equation with respect to $z$ :

$$
\frac{\partial \Phi}{\partial z}=A \Phi=\sum_{i=0}^{N+1} \frac{A_{i}}{z-u_{i}} \Phi
$$

where $u_{N+1}=0$ and the $L \times L$ matrices $A_{i}$ read

$$
\begin{aligned}
A_{i} & =-\left(\begin{array}{cccc}
0 & v_{0,1}^{(i)} & \cdots & v_{0, L-1}^{(i)} \\
& 0 & \ddots & \vdots \\
& & \ddots & v_{L-2, L-1}^{(i)} \\
& & & 0
\end{array}\right)-u_{i}\left(\begin{array}{cccc}
v_{0,0}^{(i)} & & & O \\
v_{1,0}^{(i)} & v_{1,1}^{(i)} & & \\
\vdots & & \ddots & \\
v_{L-1,0}^{(i)} & v_{L-1,1}^{(i)} & \cdots & v_{L-1, L-1}^{(i)}
\end{array}\right) \quad(0 \leq i \leq N), \\
A_{N+1} & =\left(\begin{array}{cccc}
e_{0} & w_{0,1} & \cdots & w_{0, L-1} \\
& e_{1} & \ddots & \vdots \\
& & \ddots & w_{L-2, L-1} \\
& & &
\end{array}\right)
\end{aligned}
$$

with

$$
e_{n}=\frac{d_{n,-n-1}-d_{n-1,-n-1}+n}{L} \quad \text { and } \quad w_{m, n}=\sum_{i=0}^{N} v_{m, n}^{(i)} .
$$

The linear differential equation (6.8) is Fuchsian and has the $N+3$ regular singularities $u_{0}, u_{1}, \ldots, u_{N}$, $u_{N+1}=0, u_{N+2}=\infty$. Observe that every $A_{i}(0 \leq i \leq N)$ is not full rank unlike $A_{N+1}$ and 
$A_{N+2}=-\sum_{i=0}^{N+1} A_{i}$. To be specific, if we prepare the column vector $\boldsymbol{b}^{(i)}$ and the row vector $\boldsymbol{c}^{(i)}$ defined by

$$
\begin{aligned}
{ }^{\mathrm{T}} \boldsymbol{b}^{(i)} & =\left(\frac{-g_{n,-n}^{(i)}}{L t_{i}{ }^{n} \prod_{m=1}^{n} f_{m,-m+1}^{(i)}}\right)_{0 \leq n \leq L-1}=\frac{-1}{L}\left(g_{0,0}^{(i)}, \frac{g_{1,-1}^{(i)}}{t_{i} f_{1,0}^{(i)}}, \frac{g_{2,-2}^{(i)}}{t_{i}{ }^{2} f_{1,0}^{(i)} f_{2,-1}^{(i)}}, \ldots, \frac{g_{L-1,-L+1}^{(i)}}{t_{i}{ }^{L-1} f_{1,0}^{(i)} f_{2,-1}^{(i)} \cdots f_{L-1,2}^{(i)}}\right), \\
\boldsymbol{c}^{(i)} & =\left(t_{i}{ }^{n} \prod_{m=1}^{n} f_{m,-m+1}^{(i)}\right)_{0 \leq n \leq L-1}=\left(1, t_{i} f_{1,0}^{(i)}, t_{i}^{2} f_{1,0}^{(i)} f_{2,-1}^{(i)}, \ldots, t_{i}^{L-1} f_{1,0}^{(i)} f_{2,-1}^{(i)} \cdots f_{L-1,2}^{(i)}\right),
\end{aligned}
$$

then we have indeed

$$
A_{i}=\boldsymbol{b}^{(i)} \cdot \boldsymbol{c}^{(i)} \quad \text { and } \quad \boldsymbol{c}^{(i)} \cdot \boldsymbol{b}^{(i)}=-\sum_{n=0}^{L-1} \frac{g_{n,-n}^{(i)}}{L}=-\theta_{i} \in \mathbb{C}
$$

for $0 \leq i \leq N$. The matrix $A_{N+2}=-\sum_{i=0}^{N+1} A_{i}$ is lower triangular and its diagonal entries are

$$
\sum_{i=0}^{N} u_{i} v_{n, n}^{(i)}-e_{n}=\sum_{i=0}^{N} \frac{g_{n,-n}^{(i)}}{L}-e_{n}=\kappa_{n}-e_{n}
$$

for $0 \leq n \leq L-1$. Here we have used (6.5) and (6.7) and put

$$
\kappa_{n}=\frac{\kappa_{n,-n}}{L}=\frac{d_{n,-n-1}-d_{n-1,-n}+\sum_{i=0}^{N} \theta_{i}}{L}=\sum_{i=0}^{N} \frac{g_{n,-n}^{(i)}}{L}
$$

cf. (4.13). Hence the characteristic exponents of (6.8) at each singularity $z=u_{i}$, i.e., the eigenvalues of each residue matrix $A_{i}$, are listed as follows:

\begin{tabular}{cc}
\hline Singularity & Exponents \\
\hline$u_{i}(0 \leq i \leq N)$ & $\left(-\theta_{i}, 0, \ldots, 0\right)$ \\
$u_{N+1}=0$ & $\left(e_{0}, e_{1}, \ldots, e_{L-1}\right)$ \\
$u_{N+2}=\infty$ & $\left(\kappa_{0}-e_{0}, \kappa_{1}-e_{1}, \ldots, \kappa_{L-1}-e_{L-1}\right)$ \\
\hline
\end{tabular}

Note that the relations

$$
\sum_{n=0}^{L-1} e_{n}=\frac{L-1}{2} \text { and } \sum_{n=0}^{L-1} \kappa_{n}=\sum_{i=0}^{N} \theta_{i}
$$

hold among the exponents. The sum of all the exponents certainly equals zero (Fuchs relation).

Compatibility between the above two linear equations, (6.6) and (6.8), is a priori established because both originate from the same bilinear equation (1.3). The former, (6.6), governs the monodromy preserving deformation of the latter, (6.8), along a deformation parameter $u_{i}$. The nonlinear system $\mathcal{G}_{L, N},(4.12)$, can be recovered from the integrability condition $\left[\frac{\partial}{\partial u_{i}}-B_{i}, \frac{\partial}{\partial z}-A\right]=0$ of the linear system (6.6) and (6.8).

Remark 6.2. In general, we can associate with an $L \times L$ Fuchsian system

$$
\frac{\partial \Phi}{\partial z}=A \Phi=\sum_{i=0}^{N+1} \frac{A_{i}}{z-u_{i}} \Phi
$$


having $N+3$ regular singularities $u_{0}, u_{1}, \ldots, u_{N}, u_{N+1}=0, u_{N+2}=\infty$ an $(N+3)$-tuple

$$
\mathcal{M}=\left\{\left(\mu_{0,1}, \mu_{0,2}, \ldots, \mu_{0, \ell_{0}}\right),\left(\mu_{1,1}, \mu_{1,2}, \ldots, \mu_{1, \ell_{1}}\right), \ldots,\left(\mu_{N+2,1}, \mu_{N+2,2}, \ldots, \mu_{N+2, \ell_{N+2}}\right)\right\}
$$

of partitions of $L$ in such a way that each residue matrix $A_{i}$ has the eigenvalues of multiplicity $\mu_{i, j}$; we call $\mathcal{M}$ the spectral type. The number of accessory parameters of (6.13) is known to be an even given by

$$
(N+1) L^{2}-\sum_{i=0}^{N+2} \sum_{j=1}^{\ell_{i}} \mu_{i, j}^{2}+2 .
$$

We turn now to our case. The spectral type of (6.8) reads the $(N+3)$-tuple

$$
\underbrace{(L-1,1), \ldots,(L-1,1)}_{N+1},(1,1, \ldots, 1),(1,1, \ldots, 1)
$$

of partitions of $L$, according to the table (6.11). Applying the above formula we find the number of accessory parameters to be $2 N(L-1)$, which certainly equals the essential dimension of the phase space of $\mathcal{G}_{L, N}$ as was calculated in Sect. 4.

Remark 6.3. Thanks to the algorithm proposed by Oshima [Osh08], Fuchsian systems of the form (6.13) with a fixed number $p$ of accessory parameters can be classified by the spectral types. Let us here take our interest in the Fuchsian systems that have four or more singularities because they admit the monodromy preserving deformations. If $p=2$ then we have a single fundamental system whose spectral type is $\left\{(1,1)^{4}\right\}=\{(1,1),(1,1),(1,1),(1,1)\}$; and its deformation equation turns out to be $P_{\mathrm{VI}}\left(=G_{2,1}\right)$. If $p=4$ then the result is the four Fuchsian systems specified by the spectral types $\left\{(1,1)^{5}\right\},\left\{(2,1)^{2},(1,1,1)^{2}\right\},\left\{(3,1),(2,2)^{2},(1,1,1,1)\right\}$, and $\left\{(2,2)^{3},(2,1,1)\right\}$. The first one has two deformation parameters and it corresponds to the Garnier system in two variables. The other three cases produce nonlinear ordinary differential equations of fourth order, which have been investigated by Sakai [Sak08] as candidates of the master equations, like $P_{\mathrm{VI}}$, among the family of fourth-order Painlevé equations; he clarified the polynomial Hamiltonian structure and coalescence diagram for each. Note that the first and second of the three are equivalent respectively to $\mathcal{G}_{3,1}$ (see Example 7.3) and to the fourth-order Painlevé equation of type $D_{6}^{(1)}$ introduced by Sasano [Sas06] (see also [FS08]).

\section{Polynomial Hamiltonian structure}

In this section we present Hamiltonian formalism for the system $\mathcal{G}_{L, N}$ such that Hamiltonian functions are polynomials in the canonical variables.

The Schlesinger system is the following system of nonlinear differential equations (see [JMU81, Sch12]):

$$
\frac{\partial A_{i}}{\partial u_{i}}=-\sum_{j \neq i} \frac{\left[A_{i}, A_{j}\right]}{u_{i}-u_{j}}, \quad \frac{\partial A_{i}}{\partial u_{j}}=\frac{\left[A_{i}, A_{j}\right]}{u_{i}-u_{j}} \quad(i \neq j)
$$

for $L \times L$ matrix-valued unknown functions $A_{i}$, which describes the monodromy preserving deformations of a Fuchsian system of the form (6.13). Needless to say, $\mathcal{G}_{L, N}$ is equivalent to a particular case of the Schlesinger systems specified by the spectral type (6.14). 
Recall first that (7.1) can be written as a Hamiltonian system (see, e.g., [Man99])

$$
\frac{\partial A_{i}}{\partial u_{j}}=\left\{A_{i}, K_{j}\right\}
$$

with the Hamiltonian functions

$$
K_{i}=\frac{1}{2} \operatorname{Res}_{z=u_{i}} \operatorname{tr} A^{2}=\sum_{j \neq i} \frac{\operatorname{tr}\left(A_{i} A_{j}\right)}{u_{i}-u_{j}},
$$

where the Poisson bracket $\{$,$\} is given in a standard way by$

$$
\left\{\left(A_{i}\right)_{m, n},\left(A_{j}\right)_{m^{\prime}, n^{\prime}}\right\}=\delta_{i, j}\left(\delta_{m, n^{\prime}}\left(A_{i}\right)_{m^{\prime}, n}-\delta_{m^{\prime}, n}\left(A_{j}\right)_{m, n^{\prime}}\right) .
$$

Moreover, a method to construct canonical variables for the above Hamiltonian system has been established; see [JMMS80, Appendix 5]. Set $A_{i}=B^{(i)} C^{(i)}$ and define a Poisson bracket $\{$,$\} over$ the space of matrices $B^{(i)}$ and $C^{(i)}$ by

$$
\left\{\left(B^{(i)}\right)_{m, n},\left(C^{(i)}\right)_{n, m}\right\}=1 \quad \text { and } \quad\{\text { otherwise }\}=0 .
$$

This Poisson bracket coincides with the previous one (7.3), in fact. Hence the Schlesinger system is equivalent to the canonical Hamiltonian system attached with the fundamental 2-form

$$
\Gamma=\sum_{i=0}^{N+1} \operatorname{tr}\left(\mathrm{d} C^{(i)} \wedge \mathrm{d} B^{(i)}\right)-\sum_{i=0}^{N+1} \mathrm{~d} K_{i} \wedge \mathrm{d} u_{i} .
$$

However, the above choice of canonical variables is redundant because it is possible to reduce the number of canonical variables to that of accessory parameters of the Fuchsian system (6.13).

Next we shall consider the Hamiltonian formalism of $\mathcal{G}_{L, N}$ and carry out the reduction of canonical variables. In this case the fundamental 2-form reads (see Sect. 6)

$$
\Gamma=\sum_{i=0}^{N} \sum_{n=0}^{L-1} \mathrm{~d} c_{n}^{(i)} \wedge \mathrm{d} b_{n}^{(i)}-\sum_{i=1}^{N} \mathrm{~d} K_{i} \wedge \mathrm{d} u_{i}
$$

with

$$
b_{n}^{(i)}=\frac{-g_{n,-n}^{(i)}}{L t_{i}{ }^{n} \prod_{m=1}^{n} f_{m,-m+1}^{(i)}} \quad \text { and } \quad c_{n}^{(i)}=t_{i}^{n} \prod_{m=1}^{n} f_{m,-m+1}^{(i)}
$$

for $0 \leq i \leq N$ and $0 \leq n \leq L-1$. Here we have fixed $t_{0}=1$ and thereby $u_{0}=1$. Observe that

$$
b_{n}^{(0)} c_{n}^{(0)}=-\kappa_{n}-\sum_{i=1}^{N} b_{n}^{(i)} c_{n}^{(i)}
$$

which follows from (6.10) by means of $b_{n}^{(i)} c_{n}^{(i)}=-g_{n,-n}^{(i)} / L$. Accordingly the first term of (7.4) can 
be computed as follows:

$$
\begin{aligned}
\sum_{i=0}^{N} \sum_{n=0}^{L-1} \mathrm{~d} c_{n}^{(i)} \wedge \mathrm{d} b_{n}^{(i)} & =\sum_{i=0}^{N} \sum_{n=1}^{L-1} \mathrm{~d} c_{n}^{(i)} \wedge \mathrm{d} b_{n}^{(i)}, \quad \text { since } c_{0}^{(i)}=1, \\
& =\sum_{i=0}^{N} \sum_{n=1}^{L-1} \mathrm{~d} \log c_{n}^{(i)} \wedge \mathrm{d}\left(b_{n}^{(i)} c_{n}^{(i)}\right) \\
& =\sum_{i=1}^{N} \sum_{n=1}^{L-1} \mathrm{~d} \log \frac{c_{n}^{(i)}}{c_{n}^{(0)}} \wedge \mathrm{d}\left(b_{n}^{(i)} c_{n}^{(i)}\right), \quad \text { using (7.5), } \\
& =\sum_{i=1}^{N} \sum_{n=1}^{L-1} \mathrm{~d}\left(\frac{c_{n}^{(i)}}{c_{n}^{(0)}}\right) \wedge \mathrm{d}\left(b_{n}^{(i)} c_{n}^{(0)}\right) \\
& =\sum_{i=1}^{N} \sum_{n=1}^{L-1} \mathrm{~d}\left(-b_{n}^{(i)} c_{n}^{(0)}\right) \wedge \mathrm{d}\left(\frac{c_{n}^{(i)}}{c_{n}^{(0)}}\right) .
\end{aligned}
$$

Let us now introduce the canonical variables $q_{n}^{(i)}$ and $p_{n}^{(i)}(1 \leq i \leq N ; 1 \leq n \leq L-1)$ defined by

$$
q_{n}^{(i)}=\frac{c_{n}^{(i)}}{c_{n}^{(0)}}, \quad p_{n}^{(i)}=-b_{n}^{(i)} c_{n}^{(0)},
$$

whose number, $2 N(L-1)$, is just enough for the Hamiltonian system under consideration; see Remark 6.2. In addition we take the change of independent variables

$$
s_{i}=\frac{1}{u_{i}}=t_{i}^{L}
$$

so that the resulting Hamiltonian function

$$
H_{i}=-\frac{K_{i}}{s_{i}^{2}}=-\frac{\operatorname{tr}\left(A_{i} A_{N+1}\right)}{s_{i}}+\sum_{\substack{j=0 \\ j \neq i}}^{N} \frac{s_{j} \operatorname{tr}\left(A_{i} A_{j}\right)}{s_{i}\left(s_{i}-s_{j}\right)}
$$

becomes identical with the standard one of $P_{\mathrm{VI}}$ when $(L, N)=(2,1)$; see Example 7.3. The fundamental 2-form is then rewritten as

$$
\Gamma=\sum_{i=1}^{N}\left(\sum_{n=1}^{L-1} \mathrm{~d} p_{n}^{(i)} \wedge \mathrm{d} q_{n}^{(i)}-\mathrm{d} H_{i} \wedge \mathrm{d} s_{i}\right) .
$$

For convenience we extendedly use the symbols $q_{n}^{(i)}$ and $p_{n}^{(i)}$ also for $i=0$ or $n=0$; namely, we put

$$
\begin{aligned}
& q_{n}^{(0)}=1, \quad p_{n}^{(0)}\left(=-b_{n}^{(0)} c_{n}^{(0)}\right)=\kappa_{n}-\sum_{i=1}^{N} q_{n}^{(i)} p_{n}^{(i)}, \\
& q_{0}^{(i)}=1, \quad p_{0}^{(i)}\left(=-b_{0}^{(i)} c_{0}^{(i)}\right)=\theta_{i}-\sum_{n=1}^{L-1} q_{n}^{(i)} p_{n}^{(i)}
\end{aligned}
$$

by taking (6.9) and (7.5) into account. We have then the 
Lemma 7.1. It holds that

$$
\begin{aligned}
\operatorname{tr}\left(A_{i} A_{j}\right) & =\sum_{m, n=0}^{L-1} q_{m}^{(i)} p_{m}^{(j)} q_{n}^{(j)} p_{n}^{(i)}, \\
\operatorname{tr}\left(A_{i} A_{N+1}\right) & =-\sum_{n=0}^{L-1} e_{n} q_{n}^{(i)} p_{n}^{(i)}-\sum_{j=0}^{N} \sum_{0 \leq m<n \leq L-1} q_{m}^{(i)} p_{m}^{(j)} q_{n}^{(j)} p_{n}^{(i)}
\end{aligned}
$$

for $i, j=0,1, \ldots, N$.

Proof. It follows from $\left(A_{i}\right)_{m, n}=b_{m}^{(i)} c_{n}^{(i)}$ that $\operatorname{tr}\left(A_{i} A_{j}\right)=\sum_{m, n=0}^{L-1}\left(A_{i}\right)_{n, m}\left(A_{j}\right)_{m, n}=\sum_{m, n=0}^{L-1} b_{n}^{(i)} c_{m}^{(i)} b_{m}^{(j)} c_{n}^{(j)}$, which thus yields (7.8) via $b_{n}^{(i)} c_{n}^{(j)}=-p_{n}^{(i)} q_{n}^{(j)}$. The diagonal entries of $A_{i} A_{N+1}$ read

$$
\begin{array}{rr}
(0,0): & \left(e_{0} b_{0}^{(i)}+w_{0,1} b_{1}^{(i)}+w_{0,2} b_{2}^{(i)}+\cdots+w_{0, L-1} b_{L-1}^{(i)}\right) c_{0}^{(i)}, \\
(1,1): & \left(e_{1} b_{1}^{(i)}+w_{1,2} b_{2}^{(i)}+\cdots+w_{1, L-1} b_{L-1}^{(i)}\right) c_{1}^{(i)}, \\
\vdots & \vdots \\
(L-2, L-2): & \left(e_{L-2} b_{L-2}^{(i)}+w_{L-2, L-1} b_{L-1}^{(i)}\right) c_{L-2}^{(i)}, \\
(L-1, L-1): & e_{L-1} b_{L-1}^{(i)} c_{L-1}^{(i)} .
\end{array}
$$

Therefore $\operatorname{tr}\left(A_{i} A_{N+1}\right)=\sum_{n=0}^{L-1} e_{n} b_{n}^{(i)} c_{n}^{(i)}+\sum_{0 \leq m<n \leq L-1} w_{m, n} b_{n}^{(i)} c_{m}^{(i)}$. If we remember $w_{m, n}=\sum_{j=0}^{N} v_{m, n}^{(j)}=$ $-\sum_{j=0}^{N} b_{m}^{(j)} c_{n}^{(j)}$, then we find $\operatorname{tr}\left(A_{i} A_{N+1}\right)=\sum_{n=0}^{L-1} e_{n} b_{n}^{(i)} c_{n}^{(i)}-\sum_{j=0}^{N} \sum_{0 \leq m<n \leq L-1} b_{m}^{(j)} c_{n}^{(j)} b_{n}^{(i)} c_{m}^{(i)}$; thus (7.9) is verified.

By virtue of Lemma 7.1 together with (7.7), the Hamiltonian function $H_{i}$ can be explicitly expressed as a polynomial in the $2 N(L-1)$ canonical variables $q_{n}^{(i)}$ and $p_{n}^{(i)}(1 \leq i \leq N ; 1 \leq n \leq$ $L-1)$. Finally we arrive at the

Theorem 7.2. The system $\mathcal{G}_{L, N}$ is equivalent to the canonical Hamiltonian system

$$
\frac{\partial q_{n}^{(i)}}{\partial s_{j}}=\frac{\partial H_{j}}{\partial p_{n}^{(i)}}, \quad \frac{\partial p_{n}^{(i)}}{\partial s_{j}}=-\frac{\partial H_{j}}{\partial q_{n}^{(i)}}
$$

for $i, j=1,2, \ldots, N$ and $n=1,2, \ldots, L-1$, where the Hamiltonian function $H_{i}$ is defined by

$$
s_{i} H_{i}=\sum_{n=0}^{L-1} e_{n} q_{n}^{(i)} p_{n}^{(i)}+\sum_{j=0}^{N} \sum_{0 \leq m<n \leq L-1} q_{m}^{(i)} p_{m}^{(j)} q_{n}^{(j)} p_{n}^{(i)}+\sum_{\substack{j=0 \\ j \neq i}}^{N} \frac{s_{j}}{s_{i}-s_{j}} \sum_{m, n=0}^{L-1} q_{m}^{(i)} p_{m}^{(j)} q_{n}^{(j)} p_{n}^{(i)}
$$

with (7.7).

We write the constant parameters contained in (7.10) as

$$
\vec{\kappa}=\left(e_{0}, e_{1}, \ldots, e_{L-1}, \kappa_{0}, \kappa_{1}, \ldots, \kappa_{L-1}, \theta_{0}, \theta_{1}, \ldots, \theta_{N}\right),
$$

whose number is essentially $2 L+N-1$ according to (6.12). Let $\mathcal{H}_{L, N}=\mathcal{H}_{L, N}(\vec{\kappa})$ denote the Hamiltonian system (7.10). Since all the differential equations originate from a single equation (1.3), the system $\mathcal{H}_{L, N}$ is a priori completely integrable (in the Frobenius sense). Or it can be 
shown directly by noticing the following facts: (i) the 1-form $\omega=\sum_{i=1}^{N} H_{i} \mathrm{~d} s_{i}$ is closed for an arbitrary solution of (7.10); (ii) the relation

$$
\left(\frac{\partial}{\partial s_{j}}\right) H_{i}=\left(\frac{\partial}{\partial s_{i}}\right) H_{j}=\frac{1}{\left(s_{i}-s_{j}\right)^{2}} \sum_{m, n=0}^{L-1} q_{m}^{(i)} p_{m}^{(j)} q_{n}^{(j)} p_{n}^{(i)} \quad(i \neq j)
$$

holds, where the symbol $\left(\partial / \partial s_{i}\right)$ denotes the differentiation such that $q_{n}^{(i)}$ and $p_{n}^{(i)}$ are viewed to be independent of $s_{i}$. These facts imply the commutativity of the flows induced by $H_{1}, H_{2}, \ldots, H_{N}$.

The correspondence between the canonical variables $q_{n}^{(i)}$ and $p_{n}^{(i)}$ and the dependent variables given in Sect. 4 is summarized as

$$
\begin{aligned}
q_{n}^{(i)} & =\frac{c_{n}^{(i)}}{c_{n}^{(0)}}=\left(\frac{t_{i}}{t_{0}}\right)^{n} \prod_{m=1}^{n} \frac{f_{m,-m+1}^{(i)}}{f_{m,-m+1}^{(0)}}=\left(\frac{t_{i}}{t_{0}}\right)^{n} \frac{\sigma_{n,-n}\left(\theta_{i}+1\right) \sigma_{0,0}\left(\theta_{0}+1\right)}{\sigma_{0,0}\left(\theta_{i}+1\right) \sigma_{n,-n}\left(\theta_{0}+1\right)}, \\
q_{n}^{(i)} p_{n}^{(i)} & =-b_{n}^{(i)} c_{n}^{(i)}=\frac{g_{n,-n}^{(i)}}{L}=\frac{\theta_{i}}{L} \frac{\sigma_{n-1,-n-1}\left(\theta_{i}-1\right) \sigma_{n,-n}\left(\theta_{i}+1\right)}{\sigma_{n,-n-1} \sigma_{n-1,-n}} .
\end{aligned}
$$

Example 7.3 (Case $N=1$ ). Let us restrict ourselves to the case $N=1$; thus $\mathcal{H}_{L, 1}$ becomes a system of ordinary differential equations. We begin with the case $L=2$, which is the first nontrivial one. Write $(q, p, H, s)=\left(q_{1}^{(1)}, p_{1}^{(1)}, H_{1}, s_{1}\right)$ and $\theta=\theta_{1}$. Then the Hamiltonian function can be expressed as

$$
H=H_{\mathrm{VI}}\left(a_{0}, a_{1}, a_{2}, a_{3}, a_{4} ; q, p\right)+\frac{\theta\left(e_{0}(s-1)+\kappa_{0}-\theta\right)}{s(s-1)}
$$

under the substitution

$$
a_{0}=e_{0}-e_{1}+\kappa_{1}+1, \quad a_{1}=-\kappa_{1}+\theta, \quad a_{2}=-\theta, \quad a_{3}=-e_{0}+e_{1}+\kappa_{0}, \quad a_{4}=-\kappa_{0}+\theta .
$$

Here $H_{\mathrm{VI}}=H_{\mathrm{VI}}\left(a_{0}, a_{1}, a_{2}, a_{3}, a_{4} ; q, p\right)$ denotes the Hamiltonian function of $P_{\mathrm{VI}}$ and is defined by

$$
\begin{aligned}
s(s-1) H_{\mathrm{VI}}= & q(q-1)(q-s) p^{2} \\
& -\left(\left(a_{0}-1\right) q(q-1)+a_{3} q(q-s)+a_{4}(q-1)(q-s)\right) p \\
& +a_{2}\left(a_{1}+a_{2}\right) q
\end{aligned}
$$

with $a_{i}$ being constant parameters such that $a_{0}+a_{1}+2 a_{2}+a_{3}+a_{4}=1$; see [Malm22, Oka87].

Now we turn to the case of general $L \geq 2$. Let $\left(q_{n}, p_{n}, H, s\right)=\left(q_{n}^{(1)}, p_{n}^{(1)}, H_{1}, s_{1}\right)$ and $\theta=\theta_{1}$. Then the Hamiltonian function of $\mathcal{H}_{L, 1}$ takes a coupled form of $P_{\mathrm{VI}}$ ones as follows:

$$
\begin{aligned}
H= & \sum_{n=1}^{L-1} H_{\mathrm{VI}}\left(a_{0, n}, a_{1, n}, a_{2, n}, a_{3, n}, a_{4, n} ; q_{n}, p_{n}\right)+\frac{\theta\left(e_{0}(s-1)+\kappa_{0}-\theta\right)}{s(s-1)} \\
& +\sum_{1 \leq m<n \leq L-1} \frac{\left(q_{m}-1\right) p_{m} q_{n}\left(\left(q_{n}-s\right) p_{n}-\kappa_{n}\right)+\left(q_{n}-s\right) p_{n} q_{m}\left(\left(q_{m}-1\right) p_{m}-\kappa_{m}\right)}{s(s-1)},
\end{aligned}
$$

where the last term reflects an interaction and the correspondence of constant parameters reads

$$
a_{0, n}=e_{0}-e_{n}+\kappa_{n}+1, \quad a_{1, n}=-\kappa_{n}+\theta, \quad a_{2, n}=-\theta, \quad a_{3, n}=-e_{0}+e_{n}+\kappa_{0}, \quad a_{4, n}=-\kappa_{0}+\theta .
$$

Interestingly enough, as has been pointed out by Fuji and Suzuki (see [FS09]), the coupled Hamiltonian (7.14) can be derived alternatively from the deformation of a certain linear system that is not Fuchsian but has one regular and one irregular singularities; cf. Sect. 6. It is expected to exist some integral transform (like a Laplace one) between the two kinds of Lax formalism. 
Remark 7.4. We cite the recent result by Dubrovin and Mazzocco [DM07]; they have studied Hamiltonian formalism of the Schlesinger system associated with the general spectral type (cf. (6.14)). Their construction is based on a scalar differential equation of higher order that is reduced from a Fuchsian system of the form (6.13); and the apparent singularities (see [KO83]) produced by the reduction procedure are adopted as the half of the canonical variables, i.e., the generalized coordinates. The resulting Hamiltonian system has movable algebraic branch points and thereby, unlike $\mathcal{H}_{L, N}$, does not enjoy the Painlevé property (see [Malg83, Miw81]). It would be an interesting problem to transform the general Schlesinger system into a Hamiltonian form enjoying the Painlevé property whose Hamiltonian functions are polynomials in the dependent variables.

\section{Birational canonical transformations}

This section is devoted to birational symmetries of the Hamiltonian system $\mathcal{H}_{L, N}=\mathcal{H}_{L, N}(\vec{\kappa})$. Here, to be precise, a birational canonical transformation of variables $\left(q_{n}^{(i)}, p_{n}^{(i)}, s_{i}\right)$ is said to be a symmetry if it keeps the system invariant except changing the constant parameters $\vec{k}$.

First we translate the action of $\left\langle r_{k}\right\rangle$ discussed in Sect. 3 into birational canonical transformations of $\mathcal{H}_{L, N}$. Note that $\left\langle r_{k}\right\rangle$ is isomorphic to an affine Weyl group of type $A_{L-1}^{(1)}$, denoted by $W\left(A_{L-1}^{(1)}\right)$. For each $k \in \mathbb{Z} / L \mathbb{Z}$, let $r_{k}\left(\sigma_{k, n}\right)=\hat{\sigma}_{k, n}$ and $r_{k}\left(\sigma_{m, n}\right)=\sigma_{m, n}(m \neq k)$. Substitution of (4.1) and $t=t_{i}$ in (3.5) yields

$$
D_{i} \hat{\sigma}_{k, n} \cdot \sigma_{k, n}=\theta_{i} \sigma_{k-1, n}\left(\theta_{i}-1\right) \sigma_{k+1, n}\left(\theta_{i}+1\right)
$$

with the aid of (4.2). Therefore we have

$$
\sum_{i=0}^{N} t_{i} D_{i} \hat{\sigma}_{k, n} \cdot \sigma_{k, n}=\sum_{i=0}^{N} \theta_{i} t_{i} \sigma_{k-1, n}\left(\theta_{i}-1\right) \sigma_{k+1, n}\left(\theta_{i}+1\right) .
$$

In view of the homogeneity (4.4d) we conclude that

$$
\hat{\sigma}_{k, n}=\frac{1}{\alpha_{k} \sigma_{k, n}} \sum_{i=0}^{N} \theta_{i} t_{i} \sigma_{k-1, n}\left(\theta_{i}-1\right) \sigma_{k+1, n}\left(\theta_{i}+1\right) ;
$$

recall (3.1). Similarly, we deduce from (3.6) that

$$
t_{i} \sigma_{k-1, n+1}\left(\theta_{i}-1\right) \sigma_{k+1, n}-\hat{\sigma}_{k, n+1} \sigma_{k, n}\left(\theta_{i}-1\right)+\hat{\sigma}_{k, n}\left(\theta_{i}-1\right) \sigma_{k, n+1}=0 .
$$

Through (4.5) and (4.6), the action of $r_{k}$ on $\left(f_{m, n}^{(i)}, g_{m, n}^{(i)}\right)$ is determined by (8.1) and (8.2) as follows:

$$
\begin{aligned}
r_{k}\left(f_{k, n}^{(i)}\right) & =f_{k, n}^{(i)}\left(1+\frac{\alpha_{k} t_{i} f_{k+1, n-1}^{(i)}}{\sum_{j=0}^{N} t_{j} f_{k+1, n-1}^{(j)} g_{k, n-1}^{(j)}}\right), \\
r_{k}\left(f_{k+1, n-1}^{(i)}\right) & =f_{k+1, n-1}^{(i)}\left(1-\frac{\alpha_{k} t_{i} f_{k+1, n-1}^{(i)}}{\alpha_{k} t_{i} f_{k+1, n-1}^{(i)}+\sum_{j=0}^{N} t_{j} f_{k+1, n-1}^{(j)} g_{k, n-1}^{(j)}}\right), \\
r_{k}\left(g_{k, n}^{(i)}\right) & =g_{k, n}^{(i)}\left(1+\frac{\alpha_{k} t_{i} f_{k+1, n}^{(i)}}{\sum_{j=0}^{N} t_{j} f_{k+1, n}^{(j)} g_{k, n}^{(j)}}\right), \\
r_{k}\left(g_{k+1, n-1}^{(i)}\right) & =g_{k+1, n-1}^{(i)}-\frac{\alpha_{k} t_{i} f_{k+1, n}^{(i)} g_{k, n}^{(i)}}{\sum_{j=0}^{N} t_{j} f_{k+1, n}^{(j)} g_{k, n}^{(j)}},
\end{aligned}
$$


for $n \in \mathbb{Z} / L \mathbb{Z}$. It is then easy to construct the corresponding transformation of $\left(q_{n}^{(i)}, p_{n}^{(i)}\right)$ by virtue of (7.13). Moreover, as has been mentioned in Sect. 3, the system $\mathcal{H}_{L, N}$ enjoys another action $\left\langle r_{k}{ }^{\prime}\right\rangle$ of $W\left(A_{L-1}^{(1)}\right)$ associated with the root variables $\beta_{k}=-d_{m, k-1}+2 d_{m, k}-d_{m, k+1}+1$, which commutes with the previous one $\left\langle r_{k}\right\rangle$.

Next we observe that a cyclic permutation of the suffixes $\pi:\left(\sigma_{m, n}, d_{m, n}\right) \mapsto\left(\sigma_{m+1, n-1}, d_{m+1, n-1}\right)$ keeps the bilinear expression (4.4) of $\mathcal{H}_{L, N}$ invariant, and so does the interchange of suffixes $\rho:\left(\sigma_{m, n}, d_{m, n}, t_{i}\right) \mapsto\left(\sigma_{n, m},-d_{n, m}, 1 / t_{i}\right)$. These trivial symmetries can be lifted to birational canonical transformations of $\mathcal{H}_{L, N}$. Note that $\pi$ realizes a Dynkin automorphism which rotates simultaneously the two Dynkin diagrams of type $A_{L-1}^{(1)}$ and that $\rho$ represents an interchange of the two diagrams.

For notational simplicity we extend the suffix $n$ of the canonical variables $\left(q_{n}^{(i)}, p_{n}^{(i)}\right)$ and parameters $e_{n}$ and $\kappa_{n}$ for any $n \in \mathbb{Z}$ by the conditions (cf. (7.13))

$$
q_{n+L}^{(i)}=s_{i} q_{n}^{(i)}, \quad p_{n+L}^{(i)}=\frac{p_{n}^{(i)}}{s_{i}}, \quad e_{n+L}=e_{n}+1, \quad \kappa_{n+L}=\kappa_{n}
$$

We set

$$
\mathfrak{a}_{n}=\frac{\alpha_{n}}{L}=e_{n+1}-e_{n}, \quad \mathfrak{b}_{n}=\frac{\beta_{n}}{L}=e_{L-n}-e_{L-n-1}-\kappa_{L-n}+\kappa_{L-n-1}
$$

for $0 \leq n \leq L-1$. It thus holds that $\sum_{n=0}^{L-1} \mathfrak{a}_{n}=\sum_{n=0}^{L-1} \mathfrak{b}_{n}=1$.

We now state the result.

Theorem 8.1. The Hamiltonian system $\mathcal{H}_{L, N}(\vec{\kappa})$ is invariant under the birational canonical transformations $r_{n}, r_{n}{ }^{\prime}, \pi$, and $\rho(n=0,1, \ldots, L-1)$ defined as follows:

- Action on the parameters $\vec{\kappa}$.

$$
\begin{aligned}
r_{n} & : e_{n} \mapsto e_{n}+\mathfrak{a}_{n}, \quad e_{n+1} \mapsto e_{n+1}-\mathfrak{a}_{n}, \quad \kappa_{n} \mapsto \kappa_{n}+\mathfrak{a}_{n}, \quad \kappa_{n+1} \mapsto \kappa_{n+1}-\mathfrak{a}_{n} \\
r_{n}^{\prime} & : \kappa_{L-n} \mapsto \kappa_{L-n}+\mathfrak{b}_{n}, \quad \kappa_{L-n-1} \mapsto \kappa_{L-n-1}-\mathfrak{b}_{n} . \\
\pi & : e_{n} \mapsto e_{n+1}-\frac{1}{L}, \quad \kappa_{n} \mapsto \kappa_{n+1} . \\
\rho & : e_{n} \mapsto \kappa_{L-n}-e_{L-n}-\frac{\sum_{i=0}^{N} \theta_{i}}{L}+1, \quad \kappa_{n} \mapsto \kappa_{L-n} .
\end{aligned}
$$

- Action on the canonical variables $\left(q_{n}^{(i)}, p_{n}^{(i)}\right)$.

$$
r_{n}(n \neq 0):\left\{\begin{array}{l}
q_{n}^{(i)} \mapsto q_{n}^{(i)}+\frac{\mathfrak{a}_{n}\left(q_{n+1}^{(i)}-q_{n}^{(i)}\right)}{\mathfrak{a}_{n}+\sum_{j=0}^{N} q_{n+1}^{(j)} p_{n}^{(j)}}, \\
p_{n}^{(i)} \mapsto p_{n}^{(i)}\left(1+\frac{\mathfrak{a}_{n}}{\sum_{j=0}^{N} q_{n+1}^{(j)} p_{n}^{(j)}}\right) \\
p_{n+1}^{(i)} \mapsto p_{n+1}^{(i)}-\frac{\mathfrak{a}_{n} p_{n}^{(i)}}{\sum_{j=0}^{N} q_{n+1}^{(j)} p_{n}^{(j)}}
\end{array}\right.
$$




$$
\begin{aligned}
& r_{0}:\left\{\begin{array}{l}
q_{n}^{(i)} \mapsto q_{n}^{(i)}\left(1-\frac{\mathfrak{a}_{0}\left(q_{1}^{(i)}-1\right)}{\mathfrak{a}_{0} q_{1}^{(i)}+\sum_{j=0}^{N} q_{1}^{(j)} p_{0}^{(j)}}\right), \\
p_{n}^{(i)} \mapsto p_{n}^{(i)}\left(1+\frac{\mathfrak{a}_{0}\left(q_{1}^{(i)}-1\right)}{\mathfrak{a}_{0}+\sum_{j=0}^{N} q_{1}^{(j)} p_{0}^{(j)}}\right) \quad(n \neq 1), \\
p_{1}^{(i)} \mapsto\left(p_{1}^{(i)}-\frac{\mathfrak{a}_{0} p_{0}^{(i)}}{\sum_{j=0}^{N} q_{1}^{(j)} p_{0}^{(j)}}\right)\left(1+\frac{\mathfrak{a}_{0}\left(q_{1}^{(i)}-1\right)}{\mathfrak{a}_{0}+\sum_{j=0}^{N} q_{1}^{(j)} p_{0}^{(j)}}\right) .
\end{array}\right. \\
& r_{n}^{\prime}(n \neq 0):\left\{\begin{array}{l}
q_{L-n}^{(i)} \mapsto q_{L-n}^{(i)}+\frac{\mathfrak{b}_{n}\left(q_{L-n-1}^{(i)}-q_{L-n}^{(i)}\right)}{\mathfrak{b}_{n}+\sum_{j=0}^{N} q_{L-n-1}^{(j)} p_{L-n}^{(j)}}, \\
p_{L-n}^{(i)} \mapsto p_{L-n}^{(i)}\left(1+\frac{\mathfrak{b}_{n}}{\sum_{j=0}^{N} q_{L-n-1}^{(j)} p_{L-n}^{(j)}}\right), \\
p_{L-n-1}^{(i)} \mapsto p_{L-n-1}^{(i)}-\frac{\mathfrak{b}_{n} p_{L-n}^{(i)}}{\sum_{j=0}^{N} q_{L-n-1}^{(j)} p_{L-n}^{(j)}} .
\end{array}\right. \\
& \int q_{n}^{(i)} \mapsto q_{n}^{(i)}\left(1-\frac{\mathfrak{b}_{0}\left(q_{-1}^{(i)}-1\right)}{\mathfrak{b}_{0} q_{-1}^{(i)}+\sum_{j=0}^{N} q_{-1}^{(j)} p_{0}^{(j)}}\right), \\
& r_{0}^{\prime}:\left\{\begin{array}{l}
p_{n}^{(i)} \mapsto p_{n}^{(i)}\left(1+\frac{\mathfrak{b}_{0}\left(q_{-1}^{(i)}-1\right)}{\mathfrak{b}_{0}+\sum_{j=0}^{N} q_{-1}^{(j)} p_{0}^{(j)}}\right) \quad(n \neq L-1), \\
p_{L-1}^{(i)} \mapsto \frac{1}{s_{i}}\left(p_{-1}^{(i)}-\frac{\mathfrak{b}_{0} p_{0}^{(i)}}{\sum_{j=0}^{N} q_{-1}^{(j)} p_{0}^{(j)}}\right)\left(1+\frac{\mathfrak{b}_{0}\left(q_{-1}^{(i)}-1\right)}{\mathfrak{b}_{0}+\sum_{j=0}^{N} q_{-1}^{(j)} p_{0}^{(j)}}\right) .
\end{array}\right. \\
& \pi: q_{n}^{(i)} \mapsto \frac{q_{n+1}^{(i)}}{q_{1}^{(i)}}, \quad p_{n}^{(i)} \mapsto p_{n+1}^{(i)} q_{1}^{(i)} . \\
& \rho: s_{i} \mapsto \frac{1}{s_{i}}, \quad q_{n}^{(i)} \mapsto \frac{q_{L-n}^{(i)}}{s_{i}}, \quad p_{n}^{(i)} \mapsto s_{i} p_{L-n}^{(i)} .
\end{aligned}
$$

(Here we have omitted to write the action on the variables if it is trivial.) Moreover, these transformations satisfy the relations: $r_{n}{ }^{2}=\left(r_{n} r_{n \pm 1}\right)^{3}=\left(r_{n}{ }^{\prime}\right)^{2}=\left(r_{n}{ }^{\prime} r_{n \pm 1}{ }^{\prime}\right)^{3}=\pi^{L}=\rho^{2}=\mathrm{id}, \pi r_{n}=r_{n+1} \pi$, $\pi r_{n}{ }^{\prime}=r_{n-1}{ }^{\prime} \pi$, and $\rho r_{n}=r_{n}{ }^{\prime} \rho$.

Let us explore further symmetries of $\mathcal{H}_{L, N}$ besides those in Theorem 8.1. First we consider a symmetry shifting the parameter $\theta_{i}$ to $\theta_{i}-1$ at the level of the variables $f_{m, n}^{(i)}$ and $g_{m, n}^{(i)}$. It readily follows from (4.5) and (4.6) that

$$
f_{m, n}^{(i)}\left(\theta_{i}-1\right)=\frac{g_{m, n}^{(i)}}{g_{m+1, n-1}^{(i)}} f_{m+1, n}^{(i)}
$$

Combining this with (4.11) shows that

$$
f_{m, n}^{(j)}\left(\theta_{i}-1\right)=\frac{t_{i} U_{m, n}^{(i, j)} g_{m, n}^{(i)}}{t_{j} V_{m, n}^{(i, j)} g_{m+1, n-1}^{(i)}} f_{m+1, n}^{(i)} \quad(i \neq j) .
$$

We observe for $i \neq j$ that

$$
g_{m, n}^{(j)}\left(\theta_{i}-1\right)=\theta_{j} \frac{\sigma_{m-1, n-1}\left(\theta_{i}-1, \theta_{j}-1\right) \sigma_{m, n}\left(\theta_{i}-1, \theta_{j}+1\right)}{\sigma_{m, n-1}\left(\theta_{i}-1\right) \sigma_{m-1, n}\left(\theta_{i}-1\right)}
$$




$$
\begin{aligned}
& =\frac{t_{i}-t_{j}}{t_{j}} \frac{\sigma_{m-1, n-1}\left(\theta_{i}-1, \theta_{j}-1\right) \sigma_{m, n}}{\sigma_{m, n-1}\left(\theta_{i}-1\right) \sigma_{m-1, n}\left(\theta_{j}-1\right)} \times \frac{g_{m, n+1}^{(j)}}{g_{m, n+1}^{(i)}} U_{m, n+1}^{(i, j)} \\
& =\left(\frac{t_{i}}{t_{j}} \frac{\sigma_{m, n-1}\left(\theta_{j}-1\right) \sigma_{m-1, n}\left(\theta_{i}-1\right)}{\sigma_{m, n-1}\left(\theta_{i}-1\right) \sigma_{m-1, n}\left(\theta_{j}-1\right)}-1\right) \frac{g_{m, n+1}^{(j)}}{g_{m, n+1}^{(i)}} U_{m, n+1}^{(i, j)}, \quad \text { using (4.4a), } \\
& =\left(\frac{t_{i} f_{m, n+1}^{(i)}\left(\theta_{i}-1\right)}{t_{j} f_{m, n+1}^{(j)}\left(\theta_{j}-1\right)}-1\right) \frac{g_{m, n+1}^{(j)}}{g_{m, n+1}^{(i)}} U_{m, n+1}^{(i, j)}, \quad \text { using (4.5), } \\
& =\left(\frac{t_{i} f_{m+1, n+1}^{(i)} g_{m+1, n}^{(j)}}{t_{j} f_{m+1, n+1}^{(j)} g_{m+1, n}^{(i)}}-\frac{g_{m, n+1}^{(j)}}{g_{m, n+1}^{(i)}}\right) U_{m, n+1}^{(i, j)}, \quad \text { using (8.4). }
\end{aligned}
$$

By (4.13) we have

$$
g_{m, n}^{(i)}\left(\theta_{i}-1\right)=\kappa_{m, n}-1-\sum_{j \neq i} g_{m, n}^{(j)}\left(\theta_{i}-1\right) .
$$

The transformations (8.4)-(8.7) provide a symmetry of the system $\mathcal{G}_{L, N}$, (4.12), shifting the parameter $\theta_{i}$ to $\theta_{i}-1$; however, they do not naively give a symmetry of $\mathcal{H}_{L, N}$. To reach a birational canonical transformation of $\mathcal{H}_{L, N}$, we need to combine a trivial symmetry of (4.12) shifting the suffixes: $\left(f_{m, n}^{(i)}, g_{m, n}^{(i)}, d_{m, n}\right) \mapsto\left(f_{m-1, n}^{(i)}, g_{m-1, n}^{(i)}, d_{m-1, n}\right)$. As a result we obtain a symmetry $\eta_{i}$ of $\mathcal{H}_{L, N}$ which acts on the parameters as $\theta_{i} \mapsto \theta_{i}-1$ and $\mathfrak{a}_{n} \mapsto \mathfrak{a}_{n-1}$; see Theorem 8.2 below. We do not go into detail of computations.

It is easy to find a group of symmetries $\left\langle\zeta_{i j}\right\rangle\left(\simeq \widetilde{S}_{N+1}\right)$, which is generated by a permutation of the singularities $z=u_{i}=1 / s_{i}(0 \leq i \leq N)$ of the associated Fuchsian system; see Sect. 6 .

Finally we deal with a symmetry deduced from the bilinear expression of $\mathcal{H}_{L, N}$ again. Observe that (4.4) is invariant under the transformation

$$
\iota: \sigma_{m, n}=\sigma_{m, n}(\boldsymbol{\theta}, \boldsymbol{t}) \mapsto \sigma_{-m-1,-n-1}(-\boldsymbol{\theta}, \boldsymbol{t}), \quad d_{m, n} \mapsto d_{-m-1,-n-1}, \quad \theta_{i} \mapsto-\theta_{i} .
$$

Hence we have

$$
\begin{aligned}
\iota\left(q_{n}^{(i)}\right) & =\iota\left(\left(\frac{t_{i}}{t_{0}}\right)^{n} \frac{\sigma_{n,-n}\left(\theta_{i}+1\right) \sigma_{0,0}\left(\theta_{0}+1\right)}{\sigma_{0,0}\left(\theta_{i}+1\right) \sigma_{n,-n}\left(\theta_{0}+1\right)}\right), \quad \text { using (7.13a), } \\
& =\left(\frac{t_{i}}{t_{0}}\right)^{n} \frac{\sigma_{-n-1, n-1}\left(\theta_{i}-1\right) \sigma_{-1,-1}\left(\theta_{0}-1\right)}{\sigma_{-1,-1}\left(\theta_{i}-1\right) \sigma_{-n-1, n-1}\left(\theta_{0}-1\right)} \\
& =\left(\frac{t_{i}}{t_{0}}\right)^{n} \prod_{m=-n}^{-1} \frac{f_{m,-m}^{(i)}\left(\theta_{i}-1\right)}{f_{m,-m}^{(0)}\left(\theta_{0}-1\right)} \\
& =\left(\frac{t_{i}}{t_{0}}\right)^{n} \prod_{m=-n}^{-1} \frac{g_{m,-m}^{(i)} g_{m+1,-m-1}^{(0)}}{g_{m,-m}^{(0)} g_{m+1,-m-1}^{(i)}} \frac{f_{m+1,-m}^{(i)}}{f_{m+1,-m}^{(0)}}, \quad \text { using (8.4), } \\
& =\frac{g_{-n, n}^{(i)} g_{0,0}^{(0)}}{g_{-n, n}^{(0)} g_{0,0}^{(i)}} \frac{1}{q_{-n}^{(i)}} \\
= & \frac{s_{i} p_{L-n}^{(i)} p_{0}^{(0)}}{p_{L-n}^{(0)} p_{0}^{(i)}}
\end{aligned}
$$


Similarly, it follows that

$$
\begin{aligned}
\iota\left(q_{n}^{(i)} p_{n}^{(i)}\right) & =\iota\left(\frac{\theta_{i}}{L} \frac{\sigma_{n-1,-n-1}\left(\theta_{i}-1\right) \sigma_{n,-n}\left(\theta_{i}+1\right)}{\sigma_{n,-n-1} \sigma_{n-1,-n}}\right), \quad \operatorname{using}(7.13 \mathrm{~b}), \\
& =\frac{-\theta_{i}}{L} \frac{\sigma_{-n, n}\left(\theta_{i}+1\right) \sigma_{-n-1, n-1}\left(\theta_{i}-1\right)}{\sigma_{-n-1, n} \sigma_{-n, n-1}} \\
& =-q_{L-n}^{(i)} p_{L-n}^{(i)} .
\end{aligned}
$$

These formulae (8.8) and (8.9) define a birational canonical transformation of $\mathcal{H}_{L, N}$.

The above results are summed up in the

Theorem 8.2. The Hamiltonian system $\mathcal{H}_{L, N}(\vec{\kappa})$ is invariant under the birational canonical transformations $\eta_{i}, \zeta_{i j}$, and $\iota(i, j=0,1, \ldots, N ; i \neq j)$ defined as follows:

- Action on the parameters $\vec{\kappa}$.

$$
\begin{aligned}
\eta_{i} & : e_{n} \mapsto e_{n-1}+\frac{1}{L}, \quad \kappa_{n} \mapsto \kappa_{n}-e_{n}+e_{n-1}, \quad \theta_{i} \mapsto \theta_{i}-1 . \\
\zeta_{i j} & : \theta_{i} \\
\iota & \mapsto \theta_{j} . \\
e_{n} & \mapsto-e_{L-n}+1, \quad \kappa_{n} \mapsto-\kappa_{L-n}, \quad \theta_{i} \mapsto-\theta_{i} .
\end{aligned}
$$

- Action on the canonical variables $\left(q_{n}^{(i)}, p_{n}^{(i)}\right)$.

$$
\begin{aligned}
\eta_{i}:\left\{\begin{array}{l}
q_{n}^{(j)} \mapsto \eta_{i}\left(q_{n}^{(j)}\right)=\frac{\left(\sum_{m=1}^{L} p_{-m}^{(i)}\right)\left(\sum_{m=1}^{L} p_{n-m}^{(i)} q_{n-m}^{(j)}\right)}{\left(\sum_{m=1}^{L} p_{n-m}^{(i)}\right)\left(\sum_{m=1}^{L} p_{-m}^{(i)} q_{-m}^{(j)}\right)} \quad(\text { for } \forall j), \\
p_{n}^{(j)} \mapsto \eta_{i}\left(p_{n}^{(j)}\right)=\frac{1}{\eta_{i}\left(q_{n}^{(j)}\right)} \frac{s_{j}}{s_{i}-s_{j}}\left(\frac{p_{n}^{(j)}}{p_{n}^{(i)}}-\frac{p_{n-1}^{(j)}}{p_{n-1}^{(i)}}\right) \sum_{m=1}^{L} p_{n-m}^{(i)} q_{n-m}^{(j)} \quad(j \neq i), \\
p_{n}^{(i)} \mapsto \frac{1}{\eta_{i}\left(q_{n}^{(i)}\right)}\left(\kappa_{n}-e_{n}+e_{n-1}-\sum_{j \neq i} \eta_{i}\left(q_{n}^{(j)} p_{n}^{(j)}\right)\right) .
\end{array}\right. \\
\zeta_{i j}(i, j \neq 0): s_{i} \leftrightarrow s_{j}, \quad q_{n}^{(i)} \leftrightarrow q_{n}^{(j)}, \quad p_{n}^{(i)} \leftrightarrow p_{n}^{(j)} . \\
\zeta_{i 0}=\zeta_{0 i}:\left\{\begin{array}{l}
s_{i} \mapsto \frac{1}{s_{i}}, \quad s_{j} \mapsto \frac{s_{j}}{s_{i}}, \quad q_{n}^{(i)} \mapsto \frac{1}{q_{n}^{(i)}}, \quad q_{n}^{(j)} \mapsto \frac{q_{n}^{(j)}}{q_{n}^{(i)}}, \\
p_{n}^{(i)} \mapsto q_{n}^{(i)} p_{n}^{(0)}, \quad p_{n}^{(j)} \mapsto q_{n}^{(i)} p_{n}^{(j)}(j \neq i) .
\end{array}\right. \\
\iota: q_{n}^{(i)} \mapsto \frac{s_{i} p_{L-n}^{(i)} p_{0}^{(0)}}{p_{L-n}^{(0)} p_{0}^{(i)}}, \quad p_{n}^{(i)} \mapsto-\frac{q_{L-n}^{(i)} p_{0}^{(i)} p_{L-n}^{(0)}}{s_{i} p_{0}^{(0)}} .
\end{aligned}
$$

Remark 8.3. We may regard $\mathfrak{a}_{n}, \mathfrak{b}_{n}(1 \leq n \leq L-1)$ and $\theta_{i}(0 \leq i \leq N)$ as the $2 L+N-1$ constant parameters of $\mathcal{H}_{L, N}$ instead of $\vec{\kappa}$; see (7.12) and (8.3). For reference we summarize how the birational symmetries in Theorems 8.1 and 8.2 act on $\mathfrak{a}_{n}, \mathfrak{b}_{n}$, and $\theta_{i}$ below.

$$
\begin{aligned}
& r_{n}: \mathfrak{a}_{n} \mapsto-\mathfrak{a}_{n}, \mathfrak{a}_{n \pm 1} \mapsto \mathfrak{a}_{n \pm 1}+\mathfrak{a}_{n} . \\
& r_{n}^{\prime}: \mathfrak{b}_{n} \mapsto-\mathfrak{b}_{n}, \quad \mathfrak{b}_{n \pm 1} \mapsto \mathfrak{b}_{n \pm 1}+\mathfrak{b}_{n} .
\end{aligned}
$$




$$
\begin{aligned}
\pi: \mathfrak{a}_{n} & \mapsto \mathfrak{a}_{n+1}, \quad \mathfrak{b}_{n} \mapsto \mathfrak{b}_{n-1} . \\
\rho: \mathfrak{a}_{n} & \mapsto \mathfrak{b}_{n} . \\
\eta_{i}: \mathfrak{a}_{n} & \mapsto \mathfrak{a}_{n-1}, \quad \theta_{i} \mapsto \theta_{i}-1 . \\
\zeta_{i j}: \theta_{i} & \leftrightarrow \theta_{j} . \\
\iota: \mathfrak{a}_{n} & \mapsto \mathfrak{a}_{L-n}, \quad \mathfrak{b}_{n} \mapsto \mathfrak{b}_{L-n}, \quad \theta_{i} \mapsto-\theta_{i} .
\end{aligned}
$$

Recall that the groups of canonical transformations $\left\langle r_{n}\right\rangle$ and $\left\langle r_{n}{ }^{\prime}\right\rangle$ mutually commute and each of them gives a birational realization of $W\left(A_{L-1}^{(1)}\right)$.

Remark 8.4 (Additional symmetry valid for only $N=1$ ). Let us consider the case $N=1$. Write $\left(q_{n}, p_{n}, s\right)=\left(q_{n}^{(1)}, p_{n}^{(1)}, s_{1}\right)$ and $\theta=\theta_{1}$. We then find another symmetry given as follows (see also the appendix):

$$
\varphi:\left\{\begin{array}{l}
e_{0} \mapsto \kappa_{0}-e_{0}-1, \quad e_{n} \mapsto-e_{L-n} \quad(n \neq 0) \\
\kappa_{0} \mapsto \kappa_{0}, \quad \kappa_{n} \mapsto-\kappa_{L-n} \quad(n \neq 0), \quad \theta \mapsto \kappa_{0}-\theta, \\
q_{n} \mapsto \frac{s p_{L-n}}{q_{L-n} p_{L-n}-\kappa_{L-n}}, \quad p_{n} \mapsto \frac{q_{L-n}\left(\kappa_{L-n}-q_{L-n} p_{L-n}\right)}{s}
\end{array}\right.
$$

\section{A Case $L=2$ : the Garnier system}

If $L=2$ then the canonical Hamiltonian system $\mathcal{H}_{L, N}$ is equivalent to the Garnier system in $N$ variables (see [Gar12]). This fact is guaranteed by the Lax formalism given in Sect. 6. But, however, our polynomial Hamiltonian function (7.11) is different from that given in [KO84] (see also [IKSY91]). In this appendix we describe explicitly the canonical transformation between the two Hamiltonian systems.

First we concerns the general $(L, N)$ case. Define the canonical transformation $\left(q_{n}^{(i)}, p_{n}^{(i)}, H_{i}, s_{i}\right) \mapsto$ $\left(Q_{n}^{(i)}, P_{n}^{(i)}, \widetilde{H}_{i}, s_{i}\right)$ by

$$
\begin{aligned}
Q_{n}^{(i)} & =-s_{i} \frac{p_{n}^{(i)}}{p_{n}^{(0)}}\left(=-s_{i} \frac{b_{n}^{(i)}}{b_{n}^{(0)}}\right), \\
Q_{n}^{(i)} P_{n}^{(i)} & =-q_{n}^{(i)} p_{n}^{(i)}\left(=b_{n}^{(i)} c_{n}^{(i)}\right), \\
\widetilde{H}_{i} & =H_{i}-\sum_{n=1}^{L-1} \frac{q_{n}^{(i)} p_{n}^{(i)}}{s_{i}} .
\end{aligned}
$$

Clearly the new Hamiltonian function $\widetilde{H}_{i}$ becomes again a polynomial in $Q_{n}^{(i)}$ and $P_{n}^{(i)}$. This canonical transformation is, in short, derived from an interchange of the roles of $b_{n}^{(i)}$ and $c_{n}^{(i)}$ in the definition (7.6) of the canonical variables. Note that only if $N=1$ it keeps the form of the Hamiltonian function unchanged, thereby giving rise to a birational symmetry; see Remark 8.4.

Next we let $L=2$ and write the variables as $\left(Q_{1}^{(i)}, P_{1}^{(i)}\right)=\left(q_{i}, p_{i}\right)$ for $i=1,2, \ldots, N$. The 
Hamiltonian function $\widetilde{H}_{i}$ thus takes the following expression:

$$
\begin{aligned}
s_{i}\left(s_{i}-1\right) \widetilde{H}_{i} \equiv & q_{i}\left(\kappa_{1}+\sum_{j} q_{j} p_{j}\right)\left(\kappa_{1}-\theta_{0}+\sum_{j} q_{j} p_{j}\right)+s_{i} p_{i}\left(q_{i} p_{i}+\theta_{i}\right) \\
& -\sum_{j(\neq i)} R_{j i}\left(q_{j} p_{j}+\theta_{j}\right) q_{i} p_{j}-\sum_{j(\neq i)} S_{i j}\left(q_{i} p_{i}+\theta_{i}\right) q_{j} p_{i} \\
& -\sum_{j(\neq i)} R_{i j} q_{j} p_{j}\left(q_{i} p_{i}+\theta_{i}\right)-\sum_{j(\neq i)} R_{i j} q_{i} p_{i}\left(q_{j} p_{j}+\theta_{j}\right) \\
& -\left(s_{i}+1\right)\left(q_{i} p_{i}+\theta_{i}\right) q_{i} p_{i}-\left(\theta_{N+2} s_{i}+\theta_{N+1}+1\right) q_{i} p_{i}
\end{aligned}
$$

modulo some function in only $s=\left(s_{1}, \ldots, s_{N}\right)$. Here we put $R_{i j}=s_{i}\left(s_{j}-1\right) /\left(s_{j}-s_{i}\right), S_{i j}=$ $s_{i}\left(s_{i}-1\right) /\left(s_{i}-s_{j}\right), \theta_{N+1}=d_{1,0}-d_{1,1}-1 / 2, \theta_{N+2}=d_{1,1}-d_{0,1}-1 / 2$, and $\kappa_{1}=\left(\sum_{i=0}^{N+2} \theta_{i}+1\right) / 2$. The symbols $\sum_{j}$ and $\sum_{j(\neq i)}$ stand for the summation over $j=1,2, \ldots, N$ and over $j=1, \ldots, i-1, i+$ $1, \ldots, N$, respectively. This is exactly the (usual) polynomial Hamiltonian function for the Garnier system; cf. [KO84, Tsu06].

Acknowledgement. I would like to express my sincere gratitude to Hidetaka Sakai for his helpful comments on Hamiltonian structure of the Schlesinger systems and informing me of the literature [JMMS80]. I also greatly appreciate illuminating discussions with Yasuhiro Ohta and Takao Suzuki. This work was partly conducted during my stay in the Issac Newton Institute for Mathematical Sciences at the program "Discrete Integrable Systems" (2009).

\section{References}

[DJM82] Date, E., Jimbo, M., Miwa, T.: Method for generating discrete soliton equations. II. J. Phys. Soc. Japan 51, 4125-4131 (1982)

[DM07] Dubrovin, B., Mazzocco, M.: Canonical structure and symmetries of the Schlesinger equations. Comm. Math. Phys. 271, 289-373 (2007)

[FS08] Fuji, K., Suzuki, T.: Higher order Painlevé system of type $D_{2 n+2}^{(1)}$ arising from integrable hierarchy. Int. Math. Res. Not. Vol. 2008, rnm129, 21pp (2008)

[FS09] Fuji, K., Suzuki, T.: Drinfeld-Sokolov hierarchies of type $A$ and fourth order Painlevé systems. Funkcial. Ekvac. (in press)

[Gar12] Garnier, R.: Sur des équations différentielles du troisième ordre dont l'intégrale générale est uniforme et sur une classe d'équations nouvelles d'ordre supérieur dont l'intégrale générale a ses points critiques fixes. Ann. Sci. École Norm. Sup. 29, 1-126 (1912) (French)

[IKSY91] Iwasaki, K., Kimura, H., Shimomura, S., Yoshida, M.: From Gauss to Painlevé: A Modern Theory of Special Functions. Vieweg Verlag, Braunschweig (1991)

[JMMS80] Jimbo, M., Miwa, T., Môri, Y., Sato, M.: Density matrix of an impenetrable Bose gas and the fifth Painlevé transcendent. Phys. D 1, 80-158 (1980)

[JMU81] Jimbo, M., Miwa, T., Ueno, K.: Monodromy preserving deformations of linear ordinary differential equations with rational coefficients I. Phys. D 2, 306-352 (1981)

[Kac90] Kac, V. G.: Infinite Dimensional Lie Algebras. (3rd ed.) Cambridge University Press (1990) 
[KO83] Kimura, H., Okamoto, K.: On the isomonodromic deformation of linear ordinary differential equations of higher order. Funkcial. Ekvac. 26, 37-50 (1983)

[KO84] Kimura, H., Okamoto, K.: On the polynomial Hamiltonian structure of the Garnier system. J. Math. Pures Appl. 63, 129-146 (1984)

[Koi89] Koike, K.: On the decomposition of tensor products of the representations of the classical groups: By means of the universal characters. Adv. Math. 74, 57-86 (1989)

[Malg83] Malgrange, B.: Sur les déformations isomonodromiques I. Singularités régulières. Progr. Math. 37, 401-426 (1983) (French)

[Malm22] Malmquist, J.: Sur les équations différentielles du second ordre dont l'intégrale générale a ses points critiques fixes. Ark. Mat. Astr. Fys. 17, 1-89 (1922/23) (French)

[Man99] Manin, Yu. I.: Frobenius Manifolds, Quantum Cohomology and Moduli Spaces. American Mathematical Society, Providence (1999)

[Miw81] Miwa, T.: Painlevé property of monodromy preserving equations and the analyticity of $\tau$ functions. Publ. Res. Inst. Math. Sci. 17, 703-721 (1981)

[MJD00] Miwa, T., Jimbo, M., Date, E.: Solitons: Differential Equations, Symmetries and Infinite Dimensional Algebras. Cambridge University Press (2000)

[Nou04] Noumi, M.: Painlevé Equations Through Symmetry. American Mathematical Society, Providence (2004)

[Oht07] Ohta, Y.: A generalization of the discrete UC hierarchy and its reductions. In: Elaydi, S., Cushing, J., Lasser, R., Papageorgiou, V., Ruffing, A., van Assche, W., (eds.) Difference Equations, Special Functions and Orthogonal Polynomials, pp. 471-479. World Scientific, Singapore (2007)

[Oka87] Okamoto, K.: Studies on the Painlevé equations. I. Sixth Painlevé equation $P_{\mathrm{VI}}$. Ann. Mat. Pura Appl. 146, 337-381 (1987)

[Osh08] Oshima, T.: Classification of Fuchsian systems and their connection problem. Preprint: arXiv:0811.2916

[Sak08] Sakai, H.: Talk at the international conference "From Painlevé to Okamoto", June 9-13, 2008, The University of Tokyo, Japan.

[Sas06] Sasano, Y.: Higher order Painlevé equations of type $D_{l}^{(1)}$. RIMS Koukyuroku 1473, 143-163 (2006)

[Sat81] Sato, M.: Soliton equations as dynamical systems on an infinite dimensional Grassmann manifold. RIMS Koukyuroku 439, 30-46 (1981)

[Sch12] Schlesinger, L.: Über eine klasse von differentialsystemen beliebiger ordnung mit festen kritischen punkten. J. Reine Angew. Math. 141, 96-145 (1912) (German)

[Tsu04] Tsuda, T.: Universal characters and an extension of the KP hierarchy. Comm. Math. Phys. 248, 501-526 (2004)

[Tsu05a] Tsuda, T.: Universal characters, integrable chains and the Painlevé equations. Adv. Math. 197, 587-606 (2005)

[Tsu05b] Tsuda, T.: Universal characters and $q$-Painlevé systems. Comm. Math. Phys. 260, 59-73 (2005)

[Tsu06] Tsuda, T.: Toda equation and special polynomials associated with the Garnier system. Adv. Math. 206, 657-683 (2006)

[Tsu09a] Tsuda, T.: Universal character and $q$-difference Painlevé equations. Math. Ann. 345, 395-415 (2009)

[Tsu09b] Tsuda, T.: From KP/UC hierarchies to Painlevé equations. Preprint (2009) 


\section{List of MI Preprint Series, Kyushu University}

The Global COE Program

Math-for-Industry Education \& Research Hub

MI

MI2008-1 Takahiro ITO, Shuichi INOKUCHI \& Yoshihiro MIZOGUCHI

Abstract collision systems simulated by cellular automata

MI2008-2 Eiji ONODERA

The intial value problem for a third-order dispersive flow into compact almost

Hermitian manifolds

MI2008-3 Hiroaki KIDO

On isosceles sets in the 4-dimensional Euclidean space

MI2008-4 Hirofumi NOTSU

Numerical computations of cavity flow problems by a pressure stabilized characteristiccurve finite element scheme

MI2008-5 Yoshiyasu OZEKI

Torsion points of abelian varieties with values in nfinite extensions over a padic field

MI2008-6 Yoshiyuki TOMIYAMA

Lifting Galois representations over arbitrary number fields

MI2008-7 Takehiro HIROTSU \& Setsuo TANIGUCHI

The random walk model revisited

MI2008-8 Silvia GANDY, Masaaki KANNO, Hirokazu ANAI \& Kazuhiro YOKOYAMA Optimizing a particular real root of a polynomial by a special cylindrical algebraic decomposition

MI2008-9 Kazufumi KIMOTO, Sho MATSUMOTO \& Masato WAKAYAMA

Alpha-determinant cyclic modules and Jacobi polynomials 
MI2008-10 Sangyeol LEE \& Hiroki MASUDA

Jarque-Bera Normality Test for the Driving Lévy Process of a Discretely Observed Univariate SDE

MI2008-11 Hiroyuki CHIHARA \& Eiji ONODERA

A third order dispersive flow for closed curves into almost Hermitian manifolds

MI2008-12 Takehiko KINOSHITA, Kouji HASHIMOTO and Mitsuhiro T. NAKAO

On the $L^{2}$ a priori error estimates to the finite element solution of elliptic problems with singular adjoint operator

MI2008-13 Jacques FARAUT and Masato WAKAYAMA

Hermitian symmetric spaces of tube type and multivariate Meixner-Pollaczek polynomials

MI2008-14 Takashi NAKAMURA

Riemann zeta-values, Euler polynomials and the best constant of Sobolev inequality

MI2008-15 Takashi NAKAMURA

Some topics related to Hurwitz-Lerch zeta functions

MI2009-1 Yasuhide FUKUMOTO

Global time evolution of viscous vortex rings

MI2009-2 Hidetoshi MATSUI \& Sadanori KONISHI

Regularized functional regression modeling for functional response and predictors

MI2009-3 Hidetoshi MATSUI \& Sadanori KONISHI

Variable selection for functional regression model via the $L_{1}$ regularization

MI2009-4 Shuichi KAWANO \& Sadanori KONISHI

Nonlinear logistic discrimination via regularized Gaussian basis expansions

MI2009-5 Toshiro HIRANOUCHI \& Yuichiro TAGUCHII

Flat modules and Groebner bases over truncated discrete valuation rings 
MI2009-6 Kenji KAJIWARA \& Yasuhiro OHTA

Bilinearization and Casorati determinant solutions to non-autonomous $1+1$ dimensional discrete soliton equations

\section{MI2009-7 Yoshiyuki KAGEI}

Asymptotic behavior of solutions of the compressible Navier-Stokes equation around the plane Couette flow

MI2009-8 Shohei TATEISHI, Hidetoshi MATSUI \& Sadanori KONISHI

Nonlinear regression modeling via the lasso-type regularization

MI2009-9 Takeshi TAKAISHI \& Masato KIMURA

Phase field model for mode III crack growth in two dimensional elasticity

MI2009-10 Shingo SAITO

Generalisation of Mack's formula for claims reserving with arbitrary exponents for the variance assumption

MI2009-11 Kenji KAJIWARA, Masanobu KANEKO, Atsushi NOBE \& Teruhisa TSUDA Ultradiscretization of a solvable two-dimensional chaotic map associated with the Hesse cubic curve

\section{MI2009-12 Tetsu MASUDA}

Hypergeometric T -functions of the q-Painlevé system of type $E_{8}^{(1)}$

MI2009-13 Hidenao IWANE, Hitoshi YANAMI, Hirokazu ANAI \& Kazuhiro YOKOYAMA A Practical Implementation of a Symbolic-Numeric Cylindrical Algebraic Decomposition for Quantifier Elimination

MI2009-14 Yasunori MAEKAWA

On Gaussian decay estimates of solutions to some linear elliptic equations and its applications

MI2009-15 Yuya ISHIHARA \& Yoshiyuki KAGEI

Large time behavior of the semigroup on $L^{p}$ spaces associated with the linearized compressible Navier-Stokes equation in a cylindrical domain 
MI2009-16 Chikashi ARITA, Atsuo KUNIBA, Kazumitsu SAKAI \& Tsuyoshi SAWABE Spectrum in multi-species asymmetric simple exclusion process on a ring

MI2009-17 Masato WAKAYAMA \& Keitaro YAMAMOTO

Non-linear algebraic differential equations satisfied by certain family of elliptic functions

MI2009-18 Me Me NAING \& Yasuhide FUKUMOTO

Local Instability of an Elliptical Flow Subjected to a Coriolis Force

MI2009-19 Mitsunori KAYANO \& Sadanori KONISHI

Sparse functional principal component analysis via regularized basis expansions and its application

MI2009-20 Shuichi KAWANO \& Sadanori KONISHI

Semi-supervised logistic discrimination via regularized Gaussian basis expansions

MI2009-21 Hiroshi YOSHIDA, Yoshihiro MIWA \& Masanobu KANEKO

Elliptic curves and Fibonacci numbers arising from Lindenmayer system with symbolic computations

MI2009-22 Eiji ONODERA

A remark on the global existence of a third order dispersive flow into locally Hermitian symmetric spaces

MI2009-23 Stjepan LUGOMER \& Yasuhide FUKUMOTO

Generation of ribbons, helicoids and complex scherk surface in laser-matter Interactions

MI2009-24 Yu KAWAKAMI

Recent progress in value distribution of the hyperbolic Gauss map

MI2009-25 Takehiko KINOSHITA \& Mitsuhiro T. NAKAO

On very accurate enclosure of the optimal constant in the a priori error estimates for $H_{0}^{2}$-projection 
MI2009-26 Manabu YOSHIDA

Ramification of local fields and Fontaine's property (Pm)

MI2009-27 Yu KAWAKAMI

Value distribution of the hyperbolic Gauss maps for flat fronts in hyperbolic three-space

MI2009-28 Masahisa TABATA

Numerical simulation of fluid movement in an hourglass by an energy-stable finite element scheme

MI2009-29 Yoshiyuki KAGEI \& Yasunori MAEKAWA Asymptotic behaviors of solutions to evolution equations in the presence of translation and scaling invariance

MI2009-30 Yoshiyuki KAGEI \& Yasunori MAEKAWA

On asymptotic behaviors of solutions to parabolic systems modelling chemotaxis

MI2009-31 Masato WAKAYAMA \& Yoshinori YAMASAKI

Hecke's zeros and higher depth determinants

MI2009-32 Olivier PIRONNEAU \& Masahisa TABATA

Stability and convergence of a Galerkin-characteristics finite element scheme of lumped mass type

MI2009-33 Chikashi ARITA

Queueing process with excluded-volume effect

MI2009-34 Kenji KAJIWARA, Nobutaka NAKAZONO \& Teruhisa TSUDA

Projective reduction of the discrete Painlevé system of type $\left(A_{2}+A_{1}\right)^{(1)}$

MI2009-35 Yosuke MIZUYAMA, Takamasa SHINDE, Masahisa TABATA \& Daisuke TAGAMI Finite element computation for scattering problems of micro-hologram using DtN map 
MI2009-36 Reiichiro KAWAI \& Hiroki MASUDA

Exact simulation of finite variation tempered stable Ornstein-Uhlenbeck processes

MI2009-37 Hiroki MASUDA

On statistical aspects in calibrating a geometric skewed stable asset price model

MI2010-1 Hiroki MASUDA

Approximate self-weighted LAD estimation of discretely observed ergodic OrnsteinUhlenbeck processes

MI2010-2 Reiichiro KAWAI \& Hiroki MASUDA

Infinite variation tempered stable Ornstein-Uhlenbeck processes with discrete observations

MI2010-3 Kei HIROSE, Shuichi KAWANO, Daisuke MIIKE \& Sadanori KONISHI Hyper-parameter selection in Bayesian structural equation models

MI2010-4 Nobuyuki IKEDA \& Setsuo TANIGUCHI The Itô-Nisio theorem, quadratic Wiener functionals, and 1-solitons

MI2010-5 Shohei TATEISHI \& Sadanori KONISHI

Nonlinear regression modeling and detecting change point via the relevance vector machine

MI2010-6 Shuichi KAWANO, Toshihiro MISUMI \& Sadanori KONISHI

Semi-supervised logistic discrimination via graph-based regularization

MI2010-7 Teruhisa TSUDA

UC hierarchy and monodromy preserving deformation 